\title{
Intersection theory, characteristic classes, and algebro-geometric Feynman rules
}

\author{
Paolo Aluffi ${ }^{a, *}$ and Matilde Marcolli ${ }^{b}$ \\ ${ }^{a}$ Mathematics Department, Florida State University, \\ Tallahassee, FL, USA \\ ${ }^{b}$ Division of Physics, Mathematics and Astronomy, California Institute of Technology \\ Pasadena, CA, USA \\ E-mail: aluffi@math.fsu.edu, matilde@caltech.edu
}

We review the basic definitions in Fulton-MacPherson Intersection Theory and discuss a theory of 'characteristic classes' for arbitrary algebraic varieties, based on this intersection theory. We also discuss a class of graph invariants motivated by amplitude computations in quantum field theory. These 'abstract Feynman rules' are obtained by studying suitable invariants of hypersurfaces defined by the Kirchhoff-Tutte-Symanzik polynomials of graphs. We review a 'motivic' version of these abstract Feynman rules, and describe a counterpart obtained by intersection-theoretic techniques.

MathemAmplitudes 2019: Intersection Theory \& Feynman Integrals

18-20 December 2019

Padova, Italy

\footnotetext{
${ }^{*}$ Speaker
} 


\section{Introduction}

One of us had the good fortune to participate in the meeting on Intersection Theory \& Feynman Integrals held in Padova in December 2019, nicknamed MathemAmplitudes 2019. Intersection Theory is a venerable branch of Algebraic Geometry, with roots grounded in the very origin of the subject and many ramifications tracing its history to the present day. It is a vast field, and it would be futile to attempt a comprehensive review. The aspects of the theory with which we are most familiar have a different flavor from the Intersection Theory used in the impressive new approach to computations of Feynman integrals, presented in other seminars delivered in this workshop; in this paper we will limit our discussion to the 'standard' Intersection Theory in the context of scheme-theoretic Algebraic Geometry, developed by W. Fulton and R. MacPherson in the '70s and '80s. This theory provided much-needed foundations for a large gamut of applications to questions in Algebraic Geometry, for example classical enumerative questions and the study of singularities. W. Fulton's outstanding text [1] still stands as a complete and thorough account of the foundations of this theory 35 years after its publication. For a further rich selection of examples of applications of this classically inspired, but very modern, theory, the recent book [2] can be heartily recommended. Through its manifold applications, Fulton-MacPherson's intersection theory has naturally come to interact with particle physics in different ways, for example in string theory. It is hard to believe that there should not be a strong connection between this very mature theory steeped in Algebraic Geometry and the Intersection Theory deployed so successfully in the more strictly 'MathemAmplitudes' applications that were the main object of this workshop. We view this as an interesting open question: To establish a direct interpretation of the intersectiontheoretic computations of Feynman integrals in [3-5] (and others) in terms of Fulton-MacPherson intersection theory.

We will not address this question in this paper. Our main objective is to give a necessarily incomplete overview of some ideas in Fulton-MacPherson intersection theory and of a few applications of this theory, and particularly to an aspect relating to high energy physics and to Feynman integrals. These latter may seem natural candidates for a relation with the theme of the workshop, but in all honesty we do not see a direct such connection. It may simply be that Intersection Theory is vast enough to have several different manifestations in the theory of Feynman amplitudes.

The application we will discuss employs the notion of characteristic classes beyond the classical case of compact nonsingular varieties. We will summarize the theory underlying these classes (originally due to MacPherson) after a short historical prelude and a rough exposition of the ideas behind the main definition of intersection product. A review of our application of the theory to a context inspired by quantum field theory will follow these preliminaries.

The emphasis throughout will be on what is now 'classical' intersection theory. We will only mention very briefly more recent developments, such as the extension to stacks, quantum cohomology, equivariant intersection theory, and pass over in silence much more. 


\section{Intersection theory in Algebraic Geometry}

\subsection{A little history}

Questions in enumerative geometry go so far back that it is impossible to identify a 'first' such question. The problem of Apollonius (how many circles are tangent to 3 given circles?) dates back to the second century B.C.; ancient geometric results, such as the Pappus hexagon theorem can also be framed in terms close to intersection theory.

In a much more recent past, the introduction of coordinates in geometry in the XVII century led to natural questions concerning the intersection of curves in the plane. Bézout's theorem ( 1750) may be interpreted as the first concrete manifestation of the intersection ring of projective space. It states that two curves of degrees $d_{1}, d_{2}$ meeting transversally in complex projective plane must meet in exactly $d_{1} d_{2}$ points. More generally, hypersurfaces of degrees $d_{1}, \ldots, d_{n}$ in complex $n$ dimensional projective space $\mathbb{P}^{n}$, must meet in $d_{1} \cdots d_{n}$ points if they meet transversally. Modulo many subtleties, this result may be understood as signifying that intersection theory in $\mathbb{P}^{n}$ occurs in a ring isomorphic to $\mathbb{Z}[H] /\left(H^{n+1}\right)$ : a degree- $d$ hypersurface determines the class of $d H$ in this ring, and the coefficient of $H^{n}$ in the product of the classes of a selection of hypersurfaces will yield their intersection number, provided that needed transversality hypotheses are satisfied. More generally, a subvariety $V$ of $\mathbb{P}^{n}$ of codimension $r$ will determine a class $d H^{r}$ in $\mathbb{Z}[H] /\left(H^{n+1}\right)$; the coefficient $d$ is the degree of the variety; and intersecting varieties transversally may be interpreted as performing ordinary algebra in this ring.

Bézout's theorem may already be used to analyze interesting geometric questions involving e.g., the possible singularities of plane curves or the degrees of dual curves, and it leads naturally to questions in enumerative geometry. It also raises foundational questions, such as the exact definitions of intersection numbers and the role of intersection multiplicities to deal with nontransversal intersections. In the classical school, such questions were often bypassed by tools such as the 'principle of conservation of number' (which had been accepted in one form or another since the XVII century) and Chasles's 'correspondence principle': these lead to plausible results by analyzing explicit degenerations of intersection-theoretic problems to more approachable problems involving varieties in special positions.

The subject matured to an amazing degree. By 1880, Schubert had developed (by age 30!) intersection theory with a marked modern flavor, including an explicit interpretation of intersection operations as operations in a suitable ring, with hundreds of applications. Schubert calculus refers to the intersection theory of Grassmannian, which may be reduced to the intersection of Schubert varieties, expressing natural geometric conditions.

By then, intersection theory had been applied with great success to questions in enumerative geometry. The epitomizing such question is: How many smooth conics are tangent to five smooth conics in general position? The correct answer, 3,264 (cf. the title of [2]!), was obtained by Chasles in the 1860s, and requires tools going beyond Bézout's theorem: a straightforward application of Bézout's theorem to this question has to contend with the presence of 'excess intersection'; Chasles's principle of conservation of number offers an alternative.

Another such question is the determination of the number of smooth plane cubic curves that are tangent to nine lines in general position. The number is 33,616, as found by Maillard in 1872. The number of quartic plane curves tangent to 14 lines in general position $(23,011,191,144)$ was 
determined by Zeuthen in 1873 . The corresponding number for quintics or higher degree curves was unknown in the 1870s, and it is just as unknown 150 years later.

These are very impressive results, and Schubert's theory is sophisticated and extremely successful, but it was recognized that tools such as the 'principle of conservation of number' lacked rigor in their formulation. One of Hilbert's famous 1900 problems consisted of providing firm theoretical foundations for Schubert's work. This natural question motivated substantial work in the $\mathrm{XX}$ century, by a list of mathematicians that reads as a who's who in algebraic geometry, starting with Severi. Additional motivations came later in the century from the Weil conjectures, which in time made it clear that it was necessary to extend the foundations of the theory to fields of arbitrary characteristic and to the scheme-theoretic context; and from Riemann-Roch problems, originally an indispensable tool in the study of the geometry of curves, but generalized by Hirzebruch, Grothendieck, and others to an interplay between the $K$-theory of vector bundles and coherent sheaves on a scheme and its intersection group.

In the XX century, Schubert's intersection ring evolved into the so-called 'Chow ring' of a (nonsingular) variety, where intersection-theoretic computations may be performed. As a direct offspring of the principle of conservation of number, the effect of intersecting with a subvariety, or rather a 'cycle', that is, a formal linear combination of subvarieties, should not change if the cycle is moved in a family parametrized by a projective line. Fibers of such a family are said to be 'rationally equivalent'; the elements of the Chow ring are rational equivalence classes of cycle. An approach to the formalization of intersection theory can be distilled into a 'moving lemma', proving that two given cycles may be moved in their rational equivalence classes so as to intersect transversally, and that the class of their intersection is then independent of the chosen deformations. This program was carried out in the '50s, through work of Chow and others; but a sufficiently general form of the moving lemma is actually extremely challenging, and there are reasons to believe that an 'elementary' proof of the moving lemma may simply not be available (cf. [2, §0.4.9]).

Ingredients of an alternative approach were introduced in the work of several other important figures-Segre, Verdier, Kleiman, and others. In the '70s and 80s, a complete and rigorous Intersection Theory was achieved by Fulton and MacPherson. This is in the language of schemes, over fields of arbitrary characteristic, and does not require a moving lemma; in fact, by providing a well-defined intersection product, the theory offers the most direct proof of the general form of the moving lemma. Every scheme has a Chow group of cycles modulo rational equivalence, and the theory studies operations that act on these cycles. If the scheme is nonsingular, then intersecting with a cycle may itself be interpreted as one of these operations, and the Chow group acquires a natural ring structure. This recovered and unified all partial results obtained in the previous decades and offered concrete tools for computations in e.g., enumerative geometry.

It is worth pointing out that the Chow group shares features expected of a homology theory; for example, it is a covariant functor. The Chow ring of a nonsingular variety likewise behaves as a cohomology theory: it acts on the Chow group, and is a contravariant functor. In fact, when the context allows (e.g., for complex varieties), there are natural homomorphisms from the algebraic Chow theory to homology and cohomology (particularly Borel-Moore homology; cf. [1, Chapter 19]), matching the algebro-geometric intersection operations with the corresponding topological ones. For varieties admitting a cellular decomposition, such as Grassmannians or flag manifolds, this 'cycle map' is in fact an isomorphism (cf. [1, Example 19.1.11]), so in such 
case there in fact is an isomorphism between the Chow ring and cohomology. However, beyond these poster cases the situation is quite different; for example, the dimension-0 Chow group of a complex elliptic curve is infinitely generated. Further, differential forms, which are a key tool of the intersection theory employed in the 'MathemAmplitudes' applications, are not a native ingredient in the scheme-based Fulton-MacPherson intersection theory. In this theory, even Chern classes of bundles are defined without reference to differential forms; the theory is not modeled in any direct sense after Chern-Weil theory.

\subsection{Informal overview}

We recall the basic definitions, without aiming at full generality, and stressing the intuitive aspects at the price of inevitable lack of precision. The reader is referred to [1] for a complete and efficiently organized account of the theory.

Let $V$ be an algebraic variety over an algebraically closed field $k$. A cycle of dimension $k$ on $V$ is a formal linear combination of $k$-dimensional closed subvarieties of $V$, with integer coefficients; $Z_{k}(V)$ denotes the group of $k$-dimensional cycles, and we let $Z_{*}(V)$ be the direct sum $\oplus_{k \geq 0} Z_{k}(V)$. A rational function on $V$, i.e., a regular function $f: V \rightarrow \mathbb{P}^{1}$, determines a family of cycles: for all $p \in \mathbb{P}^{1}, f^{-1}(p)$ may be written as a union of codimension-1 subvarieties, taken with suitable multiplicities. We define a relation on $Z_{k}(V)$ by prescribing that two cycles $X$ and $Y$ be related if there exists a $(k+1)$-dimensional subvariety $W \subseteq V$ and a regular function $f: W \rightarrow \mathbb{P}^{1}$ such that $X=f^{-1}(0)$ and $Y=f^{-1}(\infty)$. This prescription extends to an equivalence relation on $Z_{k}(V)$, called rational equivalence. The $k$-th Chow group of $V$ is the group $A_{k}(V)$ of cycles on $V$ modulo the subgroup determined by elements of the form $\alpha-\beta$, where $\alpha, \beta \in Z_{k}(V)$ are rationally equivalent. The Chow group of $V$ is the direct sum $\oplus_{k} A_{k}(V)$.

If $\varphi: V \rightarrow V^{\prime}$ is a proper map, a push-forward $\varphi_{*}: Z_{*}(V) \rightarrow Z_{*}\left(V^{\prime}\right)$ is defined: If $[X]$ is the cycle determined by a subvariety $X$ of $V$, then $X^{\prime}:=\varphi(X)$ is a subvariety of $V^{\prime}$ (since $\varphi$ is proper), and we can let $\varphi_{*}([X])=d \cdot\left[X^{\prime}\right]$, where $d$ denotes the degree of the restriction of $\varphi$ to $X$. (Over e.g., $\mathbb{C}, d$ is the number of points in a general fiber of $\left.\varphi\right|_{X}$, or 0 if the fiber is positivedimensional.) This prescription preserves rational equivalence, so descends to a push-forward $\varphi_{*}: A_{*}(V) \rightarrow A_{*}\left(V^{\prime}\right)$. Further, it satisfies $(\varphi \circ \psi)_{*}=\varphi_{*} \circ \psi_{*}$, so it defines $A_{*}$ as a covariant functor from the category of algebraic $k$-varieties (along with proper morphisms) to abelian groups.

A contravariant pull-back is also defined, for a restricted class of morphisms. For example, if $f: W \rightarrow V$ is a flat morphism, then one can define $f^{*}([X])=\left[f^{-1}(X)\right]$ for every closed subvariety $X$ of $V$, and extending this prescription by linearity gives a homomorphism of abelian

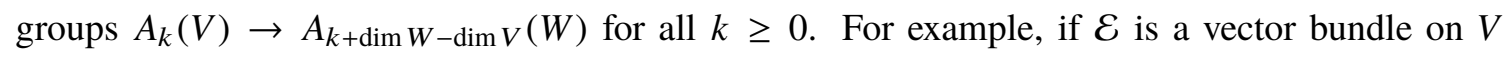
and $\pi: \mathcal{E} \rightarrow V$ denotes the projection, we have a homomorphism $\pi^{*}: A_{*}(V) \rightarrow A_{*}(\mathcal{E})$. One can prove that this is an isomorphism. (In the context of complex geometry this is perhaps not surprising, given the analogy between the Chow group and homology and the fact that the fiber of $\mathcal{E}$ are contractible. However, such considerations do not suffice, and are not needed, for the proof of this statement in the general setting of schemes.) Therefore, we may let $s^{*}: A_{*}(\mathcal{E}) \rightarrow A_{*}(V)$ be the inverse of $\pi^{*}$. This is the Gysin homomorphism, mapping $A_{*} k(\mathcal{E})$ to $A_{k-\mathrm{rk}} \mathcal{E}(V)$. As the zero-section $s: V \rightarrow \mathcal{E}$ is a right-inverse of $\pi$, it is natural to view $s^{*}$ as a pull-back of classes along the zero-section of $\mathcal{E}$, which may be identified with $V$. If $\beta \in A_{*}(\mathcal{E})$ is any rational equivalence class in $\mathcal{E}$, we can set $V \cdot \beta:=s^{*}(\beta)$ to be the 'intersection product' of $\beta$ by the zero-section in $\mathcal{E}$. 
Modulo a deformation argument, this basic intersection operation yields a very general definition of intersection product, as follows.

A subscheme of a vector bundle is a cone if it is so fiberwise. Every closed subscheme $X \subseteq V$ determines a normal cone $C_{X} V$; for example, if $X=(0, \ldots, 0)$ is the origin in affine $n$-space $\mathbb{A}^{n}$, and $V$ is the hypersurface defined by a polynomial $F\left(x_{0}, \ldots, x_{n}\right)$ vanishing at the origin, then $C_{X} V$ is the cone defined by the homogeneous terms of lowest degree in $F$. In general, the projectivization of $C_{X} V$ may be realized as the exceptional divisor in the blow-up of $V$ along $X$. A pure-dimensional subscheme $X$ is regularly embedded in $V$ if it is locally defined by $\operatorname{dim} V-\operatorname{dim} X$ equations; equivalently, if its normal cone is a vector bundle. This normal bundle is then denoted $N_{X} V$. Every closed embedding $X \subseteq V$ admits a 'deformation to the normal cone', replacing it with the embedding of $X$ as the zero-section in $C_{X} V$. Given now two subvarieties $X$ and $Y$ of $V$, assume that $X$ is regularly embedded. The embedding $X \subseteq V$ may then be deformed to the embedding of the zero-section $X \subseteq N_{X} V$; the embedding $Y \subseteq V$ is simultaneously deformed to the embedding of the cone $C_{X \cap Y} Y$ in $N_{X} V$. The modern version of the 'principle of continuity' amounts to the assertion that the intersection product $X \cdot Y$ should be preserved under such deformations. We can then define it to equal the intersection product of the zero-section with the cone $C_{X \cap Y} Y$ in the vector bundle $N_{X} V$ : as discussed above, this product admits a natural definition by means of the Gysin homomorphism.

The following diagram is a good mnemonic device for the intersection product we just introduced:

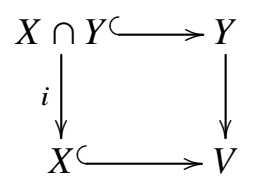

The bottom embedding is assumed to be regular, so it has a normal bundle $N_{X} V$. The intersection product $X \cdot Y \in A_{*}(V)$ may be defined as the push-forward of the intersection product of the zero-section of $N_{X} V$ with the cone $C_{X \cap Y} Y$.

Remark 2.1. In fact, $C_{X \cap Y} Y$ is naturally a subscheme of the restriction $i^{*}\left(N_{X} V\right)$ of $N_{X} V$ to the subscheme $X \cap Y \stackrel{i}{\hookrightarrow} X$; we can define $X \cdot Y$ as a class in $A_{*}(X \cap Y)$ to be the intersection product of the zero-section of this restriction with the cone $C_{X \cap Y} Y$. This definition agrees with the one sketched above after push-forward to $A_{*}(V)$, and it is often advantageous to define the product as a class in the smallest subscheme that can support it.

We also note that the definition can in fact be given for any variety $Y$ mapping to $V$, not just for closed subvarieties of $V$. For a complete discussion of the definition of intersection product, we refer the reader to [1, Chapter 6].

A more explicit 'computational' description of the intersection product introduced here will be given below.

\subsection{The intersection ring of a nonsingular variety}

The definition sketched above extends by linearity to a product of any rational equivalence class $\alpha$ in $A_{*}(V)$ by a regularly embedded subvariety $X \subseteq V$. This product is well-defined as an operator on $A_{*}(V)$, and satisfies all expected properties. 
However, this does not suffice in order to define a ring structure on the Chow group $A_{*}(V)$, mainly due to the requirement that the intersecting variety $X$ be regularly embedded in $V$. However, if $V$ is nonsingular, then there is a systematic way to reduce any intersection product to a product by a regularly embedded subvariety. The basic observation is that if $V$ is nonsingular, then the diagonal embedding $\Delta: V \rightarrow V \times V$ is a regular embedding; in fact, its normal bundle is isomorphic to the tangent bundle $T V$ of $V$. We may then consider the following intersection diagram:

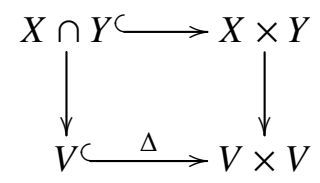

based on the observation that $X \cap Y$ is isomorphic to the scheme-theoretic intersection of the diagonal with $X \times Y$ in the product $V \times V$. In this situation we can set $X \cdot Y \in A_{*}(V)$ to be the intersection product $V \cdot(X \times Y)$ in $V \times V$, defined as discussed in $\$ 2.2$. It can be shown that this product preserves rational equivalence, and it extends by linearity to a bilinear map $A_{*}(V) \times A_{*}(V) \rightarrow A_{*}(V)$. This operation is associative and distributive with respect to the group operation in $A_{*}(V)$, hence it defines $A_{*}(V)$ as a ring.

This is the intersection (Chow) ring of the nonsingular variety $V$. A complete discussion of this construction may be found in [1, Chapter 8].

\subsection{Chern and Segre classes}

Intersection-theoretic operations arise as operators on the Chow group; above we have interpreted the intersection by a regularly embedded subvariety of $V$ as an operator on $A_{*}(V)$. As another important example, a rank- $r$ vector bundle $\mathcal{E}$ on $V$ determines 'Chern classes' $c_{i}(\mathcal{E}), i=0, \ldots, r$, as operators on $A_{*}(V)$. We write $c_{i}(\mathcal{E}) \cap \alpha$ for the result of applying the operator $c_{i}(\mathcal{E})$ to the rational equivalence class $\alpha$. The $i$-th Chern class decreases dimension by $i$ : the operator $c_{i}(\mathcal{E})$ maps $A_{k}(V)$ to $A_{k-i}(V)$. The operator $c_{0}(\mathcal{E})$ is the identity. One collects the individual Chern classes into a single operator

$$
c(\mathcal{E}):=1+c_{1}(\mathcal{E})+\cdots+c_{\mathrm{rk}} \mathcal{E}(\mathcal{E})
$$

the 'total Chern class' of $\mathcal{E}$.

If $\mathcal{E}$ is sufficiently ample, then $c_{k}(\mathcal{E}) \cap[V]$ is the class of the locus where rk $E-k+1$ general sections of $\mathcal{E}$ are linearly dependent. In particular, $c_{\mathrm{rk}} \mathcal{E}(\mathcal{E}) \cap[V]$ is the class of the zero-scheme of a regular section of $\mathcal{E}$. As a template example, if $\mathcal{L}$ is a line bundle admitting a regular section $s$, then the operator $c_{1}(\mathcal{L})$ on $A_{*}(V)$ coincides with the intersection product by the zero-scheme $D$ of $s$, a Cartier divisor of $V$ : the embedding $D \subseteq V$ is regular, so this intersection was defined in $\$ 2.2$. In other words

$$
c(\mathcal{L}) \cap \alpha=(1+D) \cdot \alpha
$$

for all $\alpha \in A_{*}(V)$. As $\mathcal{L}$ is isomorphic to the line bundle $O(D)$ determined by $D$, this discussion may be summarized by the equality

$$
c(O(D))=1+D,
$$


holding for all Cartier divisors $D$ of $V$.

In fact, Chern classes may be defined in general by means of the case of line bundles. The Segre class of a vector bundle $\mathcal{E}$ on a variety $V$ (or, more generally, a scheme) is defined as an operator on $A_{*}(V)$ by

$$
s(\mathcal{E}) \cap \alpha:=\pi_{*}\left(\sum_{i \geq 0} c(O(1))^{i} \cap \pi^{*}(\alpha)\right),
$$

where $\pi: \mathbb{P}(\mathcal{E}) \rightarrow V$ is the projective bundle (of lines) associated with $\mathcal{E}$, and $O(1)$ is the hyperplane bundle on $\mathbb{P}(\mathcal{E})$ (i.e., the dual to the tautological subbundle). One can show that $s(\mathcal{E})$ is invertible as an operator on $A_{*}(V)$, and then define

$$
c(\mathcal{E}):=s(\mathcal{E})^{-1}
$$

This notion satisfies all the expected properties of Chern classes: it is preserved by pull-backs, satisfies a projection formula $f_{*}\left(c\left(f^{*} \mathcal{E}\right) \cap \alpha\right)=c(\mathcal{E}) \cap f_{*}(\alpha)$, and the 'Whitney formula': if

$$
0 \longrightarrow \mathcal{E}^{\prime} \longrightarrow \mathcal{E} \longrightarrow \mathcal{E}^{\prime \prime} \longrightarrow 0
$$

is an exact sequence of vector bundles on $V$, then

$$
c(\mathcal{E})=c\left(\mathcal{E}^{\prime}\right) c\left(\mathcal{E}^{\prime \prime}\right)
$$

as operators on $A_{*}(V)$. The operator $c_{i}(\mathcal{E})$ equals 0 if $i>$ rk $\mathcal{E}$.

It is useful to extend the Segre class operation to more general objects. A cone $C \subseteq \mathcal{E}$ determines a subscheme $\mathbb{P}(C)$ of $\mathbb{P}(\mathcal{E})$, and this inherits by restriction a tautological bundle $O(1)$ and a projection $\pi$ to $V$. One can then define the Segre class of $C$ by

$$
s(C):=\pi_{*}\left(\sum_{i \geq 0} c(O(1))^{i} \cap[\mathbb{P}(C)]\right) .
$$

The case of the normal cone $C_{Z}(V)$ of a closed subscheme $Z \subsetneq V$ is particularly important: we define the Segre class of $Z$ in $V$ to be the Segre class of this cone:

$$
s(Z, V):=s\left(C_{Z} V\right) .
$$

(If $Z=V$, one can set $s\left(C_{Z} V\right)=[V]$.) If $Z$ is regularly embedded in $V$, so that the normal cone is the normal bundle, this definition yields

$$
s(Z, V)=c\left(N_{Z} V\right)^{-1} \cap[Z] .
$$

For example, this formula holds if $Z$ and $V$ are both nonsingular, or if $Z$ is the complete intersection of a choice of $\operatorname{codim}_{Z} V$ divisors in $V$.

If $Z=D$ is itself a Cartier divisor, then the normal bundle $N_{D} V$ is (the restriction of) $O(D)$, therefore (3) gives

$$
s(D, V)=c(O(D))^{-1} \cap[D]=(1+D)^{-1} \cdot[D]=\left(1-D+D^{2}-\cdots\right) \cdot[D] .
$$


One key property of Segre classes is their birational invariance: if $v: \widetilde{V} \rightarrow V$ is a proper birational map, then for every closed subscheme $Z$ of $V$ we have

$$
v_{*} s\left(v^{-1}(Z), \widetilde{V}\right)=s(Z, V) .
$$

In the particular case where $v: \widetilde{V} \rightarrow V$ is the blow-up of $V$ along $Z$, so that $E=v^{-1}(Z)$ is the exceptional divisor, (4) and (4) give

$$
s(Z, V)=v_{*}\left(\left(1-E+E^{2}-E^{3}+\cdots\right) \cdot[E]\right) .
$$

As $E$ is in fact isomorphic to $\mathbb{P}\left(C_{Z} V\right)$, this formula is just a restatement of the definition (2).

\subsection{The intersection product in terms of Chern and Segre classes}

The zero-section $Z$ of a vector bundle $\mathcal{E}$ on a variety $V$ is regularly embedded, and its normal bundle is $\mathcal{E}$ itself. It is useful to 'complete' the vector bundle $\mathcal{E}$ by adding a hyperplane at infinity, thereby replacing it with $\mathbb{P}\left(\mathcal{E} \oplus O_{V}\right)$. Then the 'intersection with the zero-section', i.e., the Gysin homomorphism $s^{*}$ introduced in $\S 2.2$, admits the following description. Let $\alpha \in A_{k}(\mathcal{E})$. Then

$$
s^{*}(\alpha)=\left\{c(\mathcal{E}) \cap p_{*}\left(\sum_{i \geq 0} c(O(1))^{i} \cap \bar{\alpha}\right)\right\}_{k-\mathrm{rk} \mathcal{E}}
$$

where $\{\cdots\}_{\ell}$ is the term of dimension $\ell$ in the class within braces, and $\bar{\alpha}$ is any class on $\mathbb{P}\left(\mathcal{E} \oplus O_{V}\right)$ extending $\alpha$. (This formula is a restatement of [1, Proposition 3.3].) In the situation represented by the diagram shown earlier,

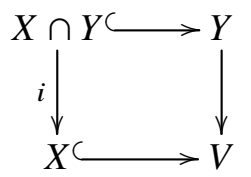

with $X, Y$ subvarieties of $V$, and $X$ regularly embedded, this formula gives the following expression for the intersection product:

$$
X \cdot Y=\left\{c\left(i^{*} N_{X} V\right) \cap s(X \cap Y, Y)\right\}_{\operatorname{dim} X+\operatorname{dim} Y-\operatorname{dim} V}
$$

([1, Proposition 6.1(a)]). If $V$ is nonsingular, then the intersection product of the rational equivalence classes of two subvarieties $X, Y$ (without further hypotheses) corresponds to the diagram

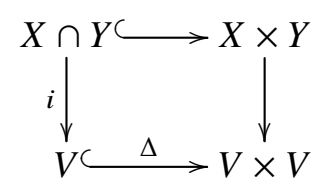

(cf. §2.3) and is given by

$$
X \cdot Y=i_{*}\left\{c\left(i^{*} T V\right) \cap s(X \cap Y, X \times Y)\right\}_{\operatorname{dim} X+\operatorname{dim} Y-\operatorname{dim} V} \in A_{*}(V),
$$

where $T V$ is the tangent bundle.

It is important to note that (7) does not require the subvarieties $X, Y$ to be in 'special position' with respect to one another. In fact, this formula is especially useful when the varieties meet with e.g., 'excess intersection', that is, when their intersection does not have the expected dimension. And again note that the formula does not 'move' the subvarieties: as stressed in $§ 2.1$, this approach to intersection theory does not rely on a moving lemma. 
Example 2.1. Let $V$ be a nonsingular compact complex algebraic variety. Its topological Euler characteristic $\chi(V)$ is then given by the 'self-intersection' $V \cdot V$ in the product $V \times V$ : indeed, according to (6), this product is given by

$$
V \cdot V=\{c(T V) \cap s(V, V)\}_{0}=c_{\operatorname{dim} V}(T V) \cap[V],
$$

and $\int c_{\operatorname{dim} V}(T V) \cap[V]=\chi(V)$ by the Poincaré-Hopf theorem (we denote by $\int \alpha$ the degree of the zero-dimensional component of a class $\alpha$ ).

Thus, we could take $V \cdot V$ as the definition of the topological Euler characteristic of $V$, and this would extend this notion to nonsingular varieties over arbitrary fields, including e.g., fields in positive characteristic.

The (degree of the) intersection in Example 2.1 is an 'ordinary' intersection number, arising from a situation which is as far from transversal as possible, and computed without deforming the intersecting varieties within their rational equivalence (or homology) classes. Fulton-MacPherson intersection theory is very successful at handling such excess intersection situations, which abound in e.g., classical enumerative problems. For example, the historically significant number 3,264 of conics tangent to 5 nonsingular conics in general position in $\mathbb{P}_{\mathbb{C}}^{2}$, mentioned in $\$ 2.1$, may be obtained using Fulton-MacPherson intersection theory by computing the relevant Segre class to evaluate directly the contribution of the excess intersection to the Bézout number. This computation is actually quite straightforward ([1, Example 9.1.9]), and extends the result to arbitrary algebraically closed fields. For example, the corresponding number over fields of characteristic 2 is 51 . A similar excess intersection technique may also be used to recover (and justify rigorously) Maillard's result computing the number 33,616 of cubics tangent to 9 lines in general position in $\mathbb{P}_{\mathbb{C}}^{2}$. (This was done in [6]. The number was also verified in [7], by different techniques. The number for quartics was verified rigorously by Vakil [[8]].)

A natural question at this point is whether the intersection numbers needed in the MathemAmplitudes applications may be interpreted in terms of Fulton-MacPherson intersection theory. If questions of excess intersection arise naturally in the context needed for these computations, formulas such as (7) would yield the most natural approach to the computation of such numbers.

\subsection{Some more recent developments}

The definitions reviewed in the past several sections date back about 40 years, and yet remain the state of the art for intersection theory in the context of schemes. The theory has been and continues to be extremely successful; Fulton's text [1] counts thousands of citations. It would be futile to either attempt to review its applications or overview directions in which intersection theory has developed in algebraic geometry in the past several decades.

Among the issues that may be closer to possible applications to MathemAmplitudes, probably the intersection theory of moduli spaces stands out; the geometry of moduli spaces of pointed rational curves appears to be especially relevant to these computations. We will just mention several themes, with an 'initial' reference that the reader may use as a seed for a more thorough bibliographic search.

In general, intersection theory on moduli spaces requires an extension of the theory beyond schemes, to stacks. This foundational work was initiated in the '80s, [9]. In the '90s, intersection 
numbers on the moduli space of curves were the object of important conjectures by Witten, proven by Kontsevich, see [10]. A description of the tautological ring of $\mathscr{M}_{g, n}$ is available through Faber's conjectures, [11]; these are still partly open, and they have been extended in several directions. The intersection theory of $\overline{\mathscr{M}}_{0, n}$ is well understood (and does not requires extensions to stacks) after work of Keel, [12]. However, subtle questions remain about this space, related to a conjecture of Fulton's, [13].

Another fascinating interaction of physics and intersection theory stemmed directly from enumerative geometry. Physicists Candelas, de la Ossa, Green, and Parkes provided a spectacular computation of the (expected) number of rational curves of a given degree on a quintic threefold, by methods that did not use standard intersection theory [14]. This work brought mirror symmetry to the full attention of mathematician, and was also important in the development of new tools such as quantum cohomology, based on the study of moduli spaces of stable maps and on GromovWitten invariants, ([15-17]). A key ingredient in the definition of these invariants is the notion of virtual fundamental class; and one approach to the treatment of this class is through excess intersection ([18]) by means analogous to (6). These enumerative questions were also one of the motivations for the development of equivariant methods in intersection theory, particularly leaning on localization formulas. A full equivariant version of intersection theory was developed in [19].

In the rest of this paper we will focus on a completely different, and much less explored, interaction between intersection theory and theoretical physics. Among the many unmentioned developments of intersection theory of the past few decades, there is a theory of characteristic classes of singular varieties. We will recall the main definitions of an important such class, and then report on an invariant constructed on the basis of this class for hypersurfaces arising in quantum field theory, specifically associated with the contribution of ('Feynman') graphs to an amplitude computation.

In a sense this is closer to the theme of MathemAmplitudes: this work was originally motivated by the attempt to understand the type of numbers that can be obtained as Feynman amplitudes in quantum field theory. On the other hand, it is also directly in contrast with the philosophy underlying MathemAmplitudes, which aims at bypassing the perturbative approach.

$\mathrm{Be}$ is as it may, this is another instance of the conceptual interaction between intersection theory and high energy physics, and we would like to advertise it in the hope that it may stimulate (even) closer interactions. We should also point out that (Fulton-MacPherson) intersection theory is part of the standard toolbox in the work of many physicists and mathematical physicists. We will mention the work of Esole and his collaborators, broadly aimed at the study of elliptic fibrations, see [20] as one example among many.

\section{Characteristic classes of singular/noncompact algebraic varieties}

\subsection{Chern classes and the Euler characteristic}

Let $V$ be a nonsingular compact algebraic variety over $\mathbb{C}$. The tangent bundle of $V$ is a natural source of important invariants of $V$, particularly by means of its Chern classes. For example, the canonical bundle of $V$ is the top exterior power of $V$, and its Chern class equals $-c_{1}(T V) \cap[V]$, the canonical divisor of $V$. This divisor class agrees with the class determined by a meromorphic 
top differential form on $V$ by taking the difference between the divisor of zeros of the form and the divisor of poles; the vanishing of this class is one of the defining properties of Calabi-Yau varieties. More generally, the Chern classes $c_{i}(T V)$ encode an obstruction to the existence of global frames of $\operatorname{dim} V-i+1$ vector fields on $V$, or (dually, and therefore up to sign), linearly independent global differential forms on $V$. In particular, the top Chern class $c_{\operatorname{dim} V}(T V)$ quantifies the obstruction to the existence of a nonvanishing tangent vector field on $V$. The precise result here was already recalled in Example 2.1:

$$
\int c_{\operatorname{dim} V}(T V) \cap[V]=\chi(V)
$$

equals the topological Euler characteristic of $V$, by the Poincaré-Hopf theorem.

The algebro-geometric point of view provides a generalization of these considerations to more general fields: for example, we can take the left-hand side of (8) as the definition of an 'Euler characteristic' for a nonsingular, complete variety: a tangent bundle is defined in this generality (for example by reference to the sheaf of differential forms, which is an inherently algebraic object), and Chern classes are also defined in general as reviewed in §2.4. This general form of the Euler characteristic has the same pleasant properties of the topological Euler characteristic in the complex, topological sense: for example, it is multiplicative in the sense that

$$
\chi(V \times W)=\chi(V) \chi(W),
$$

and it is additive in the sense that if $V$, resp., $V^{\prime}$ are complete nonsingular varieties and $Z$, resp., $Z^{\prime}$ are closed subvarieties, and $V \backslash Z \cong V^{\prime} \backslash Z^{\prime}$ are isomorphic, then

$$
\chi(V)-\chi(Z)=\chi\left(V^{\prime}\right)-\chi\left(Z^{\prime}\right)
$$

In fact, this allows us to define a consistent notion of Euler characteristic for possibly singular, possibly noncomplete varieties: if $U=V \backslash Z$, where $Z$ is a closed subvariety of a complete nonsingular variety $V$, we may set

$$
\chi(U):=\chi(V)-\chi(Z)
$$

and if $W=\amalg_{i} U_{i}$ is a possibly singular variety, written as the disjoint union of locally closed nonsingular subvarieties, we may define

$$
\chi(W):=\sum_{i} \chi\left(U_{i}\right)
$$

In the complex case, this more general notion of Euler characteristic still agrees with the topological (compactly supported) Euler characteristic.

It is natural to ask whether more general invariants of algebraic varieties satisfy these strong multiplicative and additive properties. We will present two such notions: one directly related to intersection theory, in this section; and one of a different (but ultimately also related) nature, in §4.1.

\subsection{Chern classes of noncompact/singular varieties}

For convenience we will now work over $\mathbb{C}$; the material in this subsection has a natural generalization to arbitrary algebraically closed fields of characteristic zero ([21, 22]). 
As we have recalled, the Euler characteristic of a nonsingular compact complex algebraic variety $V$ equals the degree of the zero-dimensional part of the total Chern class of the tangent bundle of $V$,

$$
c(T V) \cap[V]=\left(1+c_{1}(T V)+\cdots+c_{\operatorname{dim} V}(T V)\right) \cap[V] \in A_{*}(V) .
$$

We will call this class the 'Chern class of $V$ ' for short. It is natural to ask whether the additivity properties of the Euler characteristic may be extended to the Chern class of a variety, and in particular whether this notion of Chern class may be extended to varieties that are not necessarily nonsingular or compact.

A natural extension would associate a 'Chern class' to every constructible (integer-valued) function $\varphi$ on a variety $V$. Every constructible function may be written as a finite sum of indicator functions of closed subvarieties, with integer coefficients:

$$
\varphi=\sum_{Z \subseteq V} m_{Z} \mathbb{1}_{Z}
$$

where $m_{Z} \in \mathbb{Z}$ and $\mathbb{1}_{Z}(p)=1$ if $p \in Z, 0$ if $p \notin Z$. Denoting by $C(V)$ the abelian group of constructible integer-valued functions, 'additivity' would imply that this extended Chern class should be a homomorphism of abelian groups

$$
c_{*}: \quad C(V) \rightarrow A_{*}(V),
$$

subject to the requirement that if $i: Z \hookrightarrow V$ is the inclusion of a closed nonsingular subvariety, then

$$
c_{*}\left(\mathbb{1}_{Z}\right)=i_{*}(c(T Z) \cap[W]) \in A_{*}(V) .
$$

These additivity and normalization requirements alone do not determine a unique homomorphism $c_{*}$. A Chern-class version of the multiplicativity property of the Euler characteristic leads to a further requirement that does determine a unique homomorphism.

For this, consider the product $V \times W$ of two compact complex algebraic varieties, with projections $\pi_{V}, \pi_{W}$ to the factors. We have

$$
T(V \times W)=\pi_{V}^{*}(T V) \oplus \pi_{W}^{*}(T W),
$$

and therefore (by the Whitney formula, (1))

$$
c(T(V \times W))=\pi_{V}^{*} c(T V) \pi_{W}^{*} c(T W) .
$$

Now observe that $\pi_{W}^{*}(c(T W) \cap[W])$ consists of classes of dimension $\geq \operatorname{dim} V$; the classes of dimension $>\operatorname{dim} V$ vanish after push-forward to $V$, while

$$
\pi_{V *}\left(c_{\operatorname{dim} W}(T W) \cap[W]\right)=\left(\int c_{\operatorname{dim} W}(T W) \cap[W]\right)[V]=\chi(W)[V] .
$$

Therefore the other good functoriality property of Chern classes recalled in $\$ 2.4$ give

$$
\begin{aligned}
\pi_{V *}(c(T(V \times W)) \cap[V \times W]) & =c(T V) \cap \pi_{V *}\left(\pi_{W}^{*}(c(T W) \cap[W])\right. \\
& =\chi(W) c(T V) \cap[V] .
\end{aligned}
$$


This tells us what functoriality property we should expect from the sought-for homomorphism $c_{*}$ in (9). We may view the abelian group $C(V)$ introduced above as the value of a functor

$$
C: \quad\{\text { compact complex varieties }\} \longrightarrow\{\text { abelian groups }\} \quad ;
$$

if $f: V \rightarrow W$ is a proper morphism, we let

$$
f_{*}: \quad C(V) \rightarrow C(W)
$$

be the homomorphism determined by letting for all closed subvarieties $Z$ of $V$ and points $p \in W$

$$
f_{*}\left(\mathbb{1}_{Z}\right)(p)=\chi\left(f^{-1}(p) \cap[Z]\right)
$$

and extending this prescription to all constructible functions by linearity. It can be shown that the right-hand side in this expression is a constructible function, and that $(f \circ g)_{*}=f_{*} \circ g_{*}$. (This is ultimately because of the additivity and multiplicativity properties of the Euler characteristic.) For example,

$$
\pi_{V *}\left(\mathbb{1}_{V \times W}\right)=\chi(W) \mathbb{1}_{V},
$$

in accord with the Chern class identity (10). With this notation, we can state the following key result.

Theorem 3.1. There exists a unique natural transformation $c_{*}: C \rightarrow A_{*}$ such that if $V$ is a nonsingular variety, then

$$
c_{*}\left(\mathbb{1}_{V}\right)=c(T V) \cap[V] .
$$

This statement was conjectured in homology by Grothendieck and Deligne, and proved by MacPherson ([23]). The extension to the Chow group may be found in [1, Example 19.1.7].

We can use the natural transformation provided by Theorem 3.1 to give the following definition. For a compact variety $V$, and every locally closed $U$, we can set

$$
c_{\mathrm{SM}}(U):=c_{*}\left(\mathbb{1}_{U}\right) \in A_{*} V
$$

The normalization requirement specified in Theorem 3.1 implies that if $U=Z \stackrel{i}{\hookrightarrow} V$ is a nonsingular, compact subvariety of $V$, then

$$
c_{\mathrm{SM}}(Z)=i_{*}(c(T Z) \cap[Z])
$$

as expected. In this sense, $c_{\mathrm{SM}}(U)$ is an extension to possibly singular, possibly noncompact varieties of the notion of total Chern class of the tangent bundle. It shares additive and multiplicative properties with the Euler characteristic. For example, let $U_{1}, U_{2}$ be two locally closed subsets of $V$. Since

$$
\mathbb{1}_{U_{1} \cup U_{2}}=\mathbb{1}_{U_{1}}+\mathbb{1}_{U_{2}}-\mathbb{1}_{U_{1} \cap U_{2}}
$$

we must have

$$
c_{\mathrm{SM}}\left(U_{1} \cup U_{2}\right)=c_{\mathrm{SM}}\left(U_{1}\right)+c_{\mathrm{SM}}\left(U_{2}\right)-c_{\mathrm{SM}}\left(U_{1} \cap U_{2}\right),
$$

an 'inclusion-exclusion' formula that is often useful in concrete computations. 
Remark 3.1. As we will observe below, the degree of $c_{\mathrm{SM}}(U)$ equals its topological Euler characteristic $\chi(U)$. More generally, all the terms in $c_{\mathrm{SM}}(U)$ may be interpreted as obstructions to the existence of 'global frames of tangent vector fields', similar to the interpretation of the components of $c_{i}(T V)$ in the nonsingular case, recalled in $\$ 3.1$. If $U$ is singular, one needs to define a suitable notion extending 'tangent vector fields'. This may be done by considering vector fields that have suitably controlled ('radial') behavior near the singularities. This fundamental insight actually preceded MacPherson's work and the formulation of the Grothendieck-Deligne conjecture: as early as 1965 ([24, 25]), Marie-Hélène Schwartz had introduced radial frames and extended this general form of the Poincaré-Hopf theorem, producing Chern classes for singular varieties, in relative cohomology. Brasselet and Schwartz later proved that the Schwartz classes and the MacPherson classes agree under Alexander duality $([26,27])$. The notation $c_{\mathrm{SM}}$ is chosen as an acronym for 'Chern-Schwartz-MacPherson'.

As an example of the benefit of the functoriality property of $c_{\mathrm{SM}}$ classes, consider the constant map $\kappa: V \rightarrow p t$ of a compact variety $V$ to a point. The fact that $c_{*}$ is a natural transformation implies that the following diagram is commutative:

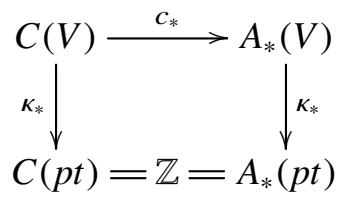

Chasing this diagram with the constructible function $\mathbb{1}_{U}$ for any locally closed subset $U \subseteq V$ :

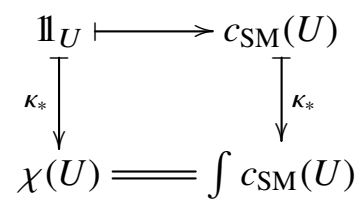

proves that

$$
\int c_{\mathrm{SM}}(U)=\chi(U)
$$

Thus, the topological Euler characteristic of $U$ equals the degree of its Chern-Schwartz-MacPherson class. This should be viewed as an extension to possibly singular, possibly noncompact varieties, of the Poincaré-Hopf theorem. The existence of a natural transformation $c_{*}$ as stated in Theorem 3.1 recovers this fundamental classical theorem as the extremely special case of constant maps on nonsingular compact varieties.

For $V=\mathbb{P}^{n}$, the CSM class of a locally closed subset $U$ is an element of $A_{*} \mathbb{P}^{n}$, that is, an integer linear combination of the classes $\left[\mathbb{P}^{0}\right], \ldots,\left[\mathbb{P}^{n}\right]$ of linear subspaces of $\mathbb{P}^{n}$ :

$$
c_{\mathrm{SM}}(U)=\sum_{i=0}^{n} a_{i}\left[\mathbb{P}^{i}\right] .
$$

The information carried by the class in this case consists of $n+1$ integers, and the foregoing considerations imply that $a_{0}=\chi(U)$ is the topological Euler characteristic of $U$. It can be shown that the integers $a_{0}, \ldots, a_{n}$ in fact determine and are determined by the Euler characteristics of the intersections of $U$ with general linear subspaces of all dimensions in $\mathbb{P}^{n}$ ([28]). 


\subsection{Effective computations and generalizations}

The definition of $c_{*}$ given by MacPherson in [23] relies on an important alternative generalization to singular varieties of the total Chern class, called the Wu-Mather Chern class, and on a subtle numerical invariant of singularities, the local Euler obstruction. In principle, the Chern class $c_{*}(\varphi)$ associated with a constructible function may be computed by using these ingredients.

In practice, however, the functoriality property of $c_{\mathrm{SM}}$ classes yields a more efficient tool for their computation. As a consequence of resolution of singularities (which holds in characteristic 0 ), for any constructible function $\varphi \in C(V)$ there exists a finite collection of nonsingular varieties $Z_{i}$ and maps $f_{i}: Z_{i} \rightarrow V$ such that

$$
\varphi=\sum_{i} m_{i} f_{i *}\left(\mathbb{1}_{Z_{i}}\right)
$$

for suitable integers $m_{i}$. Functoriality and the normalization property stated in Theorem 3.1 imply then that

$$
c_{*}(\varphi)=\sum_{i} m_{i} f_{i *}\left(c\left(T Z_{i}\right) \cap\left[Z_{i}\right]\right)
$$

In practice, this method can be refined further. Let's focus on the class $c_{S M}(V)=c_{*}\left(\mathbb{1}_{V}\right)$, where $V$ is compact but possibly singular. Decompose $V$ as a disjoint union of a collection of locally closed nonsingular subvarieties $U_{i}$ :

$$
V=\amalg_{i} U_{i}
$$

By additivity,

$$
c_{\mathrm{SM}}(V)=\sum_{i} c_{\mathrm{SM}}\left(U_{i}\right) \quad:
$$

thus, the computation of the CSM class of a variety $V$ is reduced to the computation of the contribution $c_{\mathrm{SM}}(U) \in A_{*}(V)$ of nonsingular locally closed subsets $U$. For this, consider the closure $\bar{U}$ of $U$ in $V$. Again by resolution of singularities, there exists a nonsingular complete variety $\widetilde{U}$ along with a proper map $u: \widetilde{U} \rightarrow \bar{U}$, that is an isomorphism over $U$, and such that the complement $\widetilde{U}-U$ is a divisor $D$ with normal crossings and nonsingular components $D_{i}$.

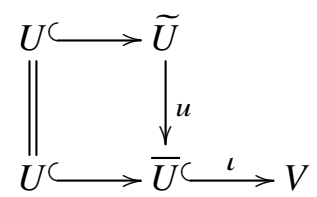

The following result computes directly the image of $c_{\mathrm{SM}}(U)$ in $A_{*}(V)$.

\section{Proposition 3.2.}

$$
c_{S M}(U)=i_{*} u_{*}\left(\frac{c(T \widetilde{U})}{\prod_{i}\left(1+D_{i}\right)} \cap[\widetilde{U}]\right)
$$

This is proved in [22], where it is also shown that this definition may be used to extend Theorem 3.1 to arbitrary algebraically closed field of characteristic 0 . The way the expression in the formula should be interpreted is as follows. As we discussed in $\$ 2.4,1+D_{i}=c\left(O\left(D_{i}\right)\right)$ is an operator on the Chow group $A_{*} V$; the intersection product by $D_{i}$ is nilpotent, since $D_{i}^{k}=0$ for 
$k>\operatorname{dim} V$; and then $1+D_{i}$ is invertible, and the notation in the 'denominator' in (13) records the action by its inverse.

Other formulas computing CSM classes bypass the use of resolution of singularities. If $X$ is a hypersurface in a nonsingular variety $V$, then

$$
c_{\mathrm{SM}}(X)=c(T V) c(O(X))^{-1} \cap\left([X]+\left(s(J X, V)^{\vee} \otimes_{V} O(X)\right)\right) .
$$

This is proved in [29]. Here $J X$ is the subscheme of $X$ defined locally by the partial derivatives of a local equation of $X$; thus, it is a scheme supported on the singular locus of $X$. The term $s(J X, V)$ denotes the Segre class introduced in $\$ 2.4$; other notation used in this statement is explained in loc. cit. A somewhat more involved statement generalizes (14) to arbitrary subschemes of nonsingular varieties, [30].

MacPherson's natural transformation and CSM classes have been generalized in several directions. Ohmoto obtained an equivariant version of the theory ([31]). The equivariant setting may be used to provide formulas for CSM classes based on localization techniques ([32]), thereby also bypassing resolution of singularities. Brasselet, Schürmann, and Yokura have introduced and studied powerful 'motivic' and associated 'Hirzebruch' classes for possibly singular varieties, depending on a parameter ([33]). For different values of the parameter, these classes may be used to recover the CSM class and other important characteristic classes (particularly the Todd class and the L class). One important feature of this approach is the role played by the Grothendieck group of algebraic varieties. In some sense this works as a replacement for the functor of constructible functions considered above. We will review the definition of this object in the next section, but will not expand on its role in the theory introduced in [33].

CSM classes (as well as the more recent generalizations mentioned in the previous paragraph) have been computed for many classical varieties; among others we mention Schubert varieties in flag manifolds, a topic that has received recent attention and revealed deep connections with e.g., the theory of stable envelopes of Maulik and Okounkov ([34], [35], and several others). The reader is warned that many important aspects of the theory, such as the role of characteristic cycles or $\mathcal{D}$-modules, are entirely omitted in the short summary sketched above. Our goal has simply been to alert the reader of the existence of these important invariants, of their 'geometric' interpretation, and of the fact that there are effective intersection-theoretic techniques to compute them if suitable information is available. In the section that follows we will use these classes to define invariants associated with graphs, motivated by the role of certain related hypersurfaces in perturbative expansions of Feynman amplitudes and by considerations stemming from computations in the Grothendieck ring of varieties.

\section{4. 'Feynman motives' and $c_{S M}$ classes of graph hypersurfaces}

\subsection{The Grothendieck ring of varieties}

In $\$ 3.2$ we have presented one substantial generalization of the Euler characteristic, with values in a Chow group. There is a different generalization, which extends the good additivity and multiplicativity properties of the Euler characteristic in an essentially tautological fashion, and which has revealed to be very useful. 
Consider the free abelian group $I\left(\operatorname{Var}_{k}\right)$ on isomorphism classes of varieties over a field $k$ (or more generally over $\mathbb{Z}$; or over a fixed scheme). We simply impose relations capturing additivity in the simplest, most general way:

$$
K\left(\operatorname{Var}_{k}\right):=I\left(\operatorname{Var}_{k}\right) /\langle[V]-[Z]-[U]\rangle \quad:
$$

here $V$ ranges over all $k$-varieties, $Z \subseteq V$ is a closed subvariety, and $U=V \backslash Z$ is the complement in $V ;\langle[V]-[Z]-[U]\rangle$ is the subgroup generated by all these elements. We can give a ring structure to the group $K\left(\operatorname{Var}_{k}\right)$ by declaring that $[V] \cdot[W]=[V \times W]$ for all varieties $V$ and $W$, and extending this definition by linearity; the identity element 1 is the class of a point. The resulting ring is the 'Grothendieck ring of $k$-varieties'.

Example 4.1. Denote by $\mathbb{L}$ the class $\left[\mathbb{A}^{1}\right]$ of the affine line in the ring $K\left(\operatorname{Var}_{k}\right)$; this is called the 'Lefschetz-Tate motive'. Then

$$
\left[\mathbb{P}_{k}^{n}\right]=\mathbb{L}^{n}+\mathbb{L}^{n-1}+\cdots+1=\frac{\mathbb{L}^{n+1}-1}{\mathbb{L}-1} \in K\left(\operatorname{Var}_{k}\right) \quad:
$$

indeed, we can write $\mathbb{P}_{k}^{n}$ as a disjoint union of affine spaces:

$$
\mathbb{P}_{k}^{n}=\mathbb{A}^{n} \amalg \mathbb{A}^{n-1} \amalg \cdots \amalg \mathbb{A}^{0}
$$

so that (15) follows from additivity in $K\left(\operatorname{Var}_{k}\right)$. (Note that $\left[\mathbb{A}^{k}\right]=[\underbrace{\mathbb{A}^{1} \times \cdots \times \mathbb{A}^{1}}_{k \text { times }}]=\mathbb{L}^{k}$.) The righthand side of (15) also records the fact that $k^{n+1} \backslash\{0\}=\mathbb{A}^{n+1} \backslash \mathbb{A}^{0}$ maps to $\mathbb{P}^{n}$ as a Zariski-locally trivial fibration with fibers $k^{*}=\mathbb{A}^{1} \backslash \mathbb{A}^{0}$.

Tautologically, every ring-valued invariant of algebraic $k$-varieties which is preserved by isomorphisms, satisfies additivity over disjoint unions, and multiplicativity over products, must factor through $K\left(\operatorname{Var}_{k}\right)$. Thus, the association of a variety $V$ with its class [V] in the Grothendieck ring $K\left(\operatorname{Var}_{k}\right)$ is a universal such invariant; it is viewed as a 'universal Euler characteristic', as the topological Euler characteristic is the simplest example of such an invariant, for $k=\mathbb{C}$. Among other invariants that must factor through $K\left(\operatorname{Var}_{k}\right)$ we mention the number of points of $V$ if $k$ is a finite field, and the class in the Grothendieck group of pure Chow motives. Because of this latter connection, the class $[V]$ in $K\left(\operatorname{Var}_{k}\right)$ may be viewed as a simplified analogue of the motive of $V$, and is also known as the 'naive motive' of $V$. The Grothendieck ring $K\left(\operatorname{Var}_{k}\right)$, or rather a completed localized version of $K\left(\operatorname{Var}_{k}\right)$, is the ring of values of the sophisticated theory of motivic integration of Denef and Loeser; see [36] for an excellent survey. Also see [37] for a useful alternative presentation of the Grothendieck ring of varieties.

The Chern-Schwartz-MacPherson class satisfies additivity and multiplicativity properties, but does not factor through the Grothendieck group: indeed, it takes values in the Chow group of the variety, so its target is not a fixed group or ring. Nevertheless, there are clear similarities linking the two theories. The theory of motivic Chern classes of Brasselet, Schürmann, and Yokura defines a natural transformation from a relative version of the Grothendieck group of varieties over a given variety $V$ to the Grothendieck group of coherent sheaves of $O_{V}$-modules, adjoined with a free variable $y$. As mentioned in $\S 3.3$, this theory generalizes the theory of Chern-Schwartz-MacPherson classes summarized in $\$ 3.2$; in this context, the relative Grothendieck group plays the role of the 
group of constructible functions. In a different direction, there is an analogue to motivic integration with values in the Chow group, which may be used to recover Chern-Schwartz-MacPherson classes, see [38].

We will not review these developments here, rather highlight the simpler observation that often the work needed to perform concrete computations in $K\left(\operatorname{Var}_{k}\right)$ will also yield results at the level of CSM classes. This is the case for the graph hypersurfaces arising in the interpretation of the contribution of a given graph to a Feynman amplitude (in perturbative massless scalar field theories) as a 'period'. We will discuss this context next, and conclude the paper with a description of an invariant defined in terms of characteristic classes of graph hypersurfaces, mimicking the invariant of these objects defined below in terms of naive motives.

\subsection{Feynman integrals and graph hypersurfaces}

Let $G$ be a (undirected) graph with $n>0$ edges. The (Kirchhoff-Tutte-Symanzik) 'graph polynomial' of $G$ is the polynomial

$$
\Psi_{G}\left(t_{1}, \ldots, t_{n}\right):=\sum_{T} \prod_{e \notin T} t_{e}
$$

where $T$ ranges over the maximal spanning forests of $G$, i.e., the unions of spanning trees or the connected components of $G$, and $t_{e}$ is a variable associated with edge $e$.

Example 4.2. The graph polynomial of the 'banana graph' with 3 edges,

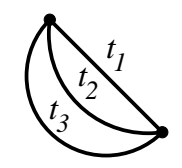

is

$$
\Psi_{G}(\underline{t})=t_{2} t_{3}+t_{1} t_{3}+t_{1} t_{2}
$$

Indeed, this graph has three spanning trees, each consisting of a single edge.

It follows immediately from the definition that $\Psi_{G}(\underline{t})$ is homogeneous, of degree equal to the number of loops in $G$. The vanishing of $\Psi_{G}(\underline{t})$ defines the 'graph hypersurface' of $G$. This may be viewed in $\mathbb{P}^{n-1}$ or as a cone in $\mathbb{A}^{n}$, where $n$ is the number of edges of $G$. The second option is preferable. In fact, the most natural object of study is the complement $\check{Y}_{G}:=\mathbb{A}^{n} \backslash \check{X}_{G}$ of the affine graph hypersurface $\check{X}_{G}$ corresponding to $G$, defined by $\Psi_{G}(\underline{t})=0$ in the ambient affine space $\mathbb{A}^{n}$.

These graph hypersurface complements $\check{Y}_{G}=\mathbb{A}^{n} \backslash \check{X}_{G}$ arise naturally in perturbative quantum field theory, when one writes the Feynman integrals for a massless scalar field theory in terms of Feynman parameters.

The physical Feynman rules for a scalar quantum field theory express the Feynman integral for a Feynman graph $G$ of the theory in the form

$$
U(G)=\int \frac{\delta\left(\sum_{e \in E_{i n t}(G)} \epsilon_{e, v} k_{e}+\sum_{e^{\prime} \in E_{e x t}(G)} \epsilon_{e^{\prime}, v} p_{e^{\prime}}\right)}{q_{1}\left(k_{1}\right) \cdots q_{n}\left(k_{n}\right)} d^{D} k_{1} \cdots d^{D} k_{n},
$$

where the constraint in the delta function is the momentum conservation relation at vertices

$$
\sum_{e \in E_{i n t}(G)} \epsilon_{e, v} k_{e}+\sum_{e^{\prime} \in E_{e x t}(G)} \epsilon_{e^{\prime}, v} p_{e^{\prime}}=0
$$


with external momenta $p_{e^{\prime}}$, with the propagators associated to edges given by the quadrics $q_{e}\left(k_{e}\right)=$ $k_{e}^{2}+m^{2}$, with $k_{e}^{2}:=\sum_{i=1}^{D} k_{e, i}^{2}$ with $D$ the spacetime dimension. The matrix $\epsilon_{e, v}$ is the incidence matrix of the graph $G$.

The "Feynman trick" or Feynman parameters representation of the Feynman integral above refers to the use of the identity

$$
\frac{1}{q_{1} \cdots q_{n}}=(n-1) ! \int_{[0,1]^{n}} \frac{\delta\left(1-\sum_{i} t_{i}\right)}{\left(t_{1} q_{1}+\cdots+t_{n} q_{n}\right)^{n}} d t_{1} \cdots d t_{n}
$$

to replace the product of quadratic forms in the denominator with a linear combination of quadratic forms and an integration of the coefficients $t_{e}$ (the Feynman parameters) over a simplex $\sigma_{n}=$ $\left\{\underline{t} \mid \sum_{i} t_{i}=1\right\}$.

One then considers a change of variables $k_{e}=u_{e}+\sum_{j=1}^{\ell} \eta_{e, j} x_{j}$, with $\eta_{e, j}$ the circuit matrix of the graph $G$ and $\ell=b_{1}(G)$ the loop number, with constraint $\sum_{e} t_{e} u_{e} \eta_{e, j}=0$, for all $j=1, \ldots, \ell$. The $u_{e}$ are taken, as a function of the external momenta, to be a solution to the equation

$$
\sum_{e \in E_{\text {int }}} \epsilon_{e, v} u_{e}+\sum_{e^{\prime} \in E_{\text {ext }}} \epsilon_{e^{\prime}, v} p_{e^{\prime}}
$$

that follows from momentum conservation at vertices and the orthogonality

$$
\sum_{e} \epsilon_{e, v} \eta_{e, j}=0
$$

for all $j=1, \ldots, \ell$ of incidence and circuit matrices. One can then take care of the integration in the $x_{i}$ variables in the resulting Feynman integral via the identity

$$
\int \frac{d^{D} x_{1} \cdots d^{D} x_{\ell}}{\left(\sum_{i} t_{i} q_{i}\right)^{n}}=C_{\ell, n} \operatorname{det}\left(M_{G}(\underline{t})\right)^{-D / 2}\left(\sum_{i} t_{i}\left(u_{i}^{2}+m^{2}\right)\right)^{-n+D \ell / 2},
$$

where $C_{\ell, n}$ is a factor independent of $G$ (for fixed number of loops and internal edges) and equal to

$$
C_{\ell, n}=\int \frac{d^{D} x_{1} \cdots d^{D} x_{n}}{\left(1+\sum_{k} x_{k}^{2}\right)^{n}}
$$

while $M_{G}(\underline{t})$ is the matrix $M_{G}(\underline{t})_{k r}=\sum_{i} t_{i} \eta_{i k} \eta_{i r}$, whose determinant is given by the graph polynomial, $\operatorname{det}\left(M_{G}(\underline{t})\right)=\Psi_{G}(\underline{t})$. One can also show that, in the massless case $m=0$, the term $\sum_{i} t_{i} u_{i}^{2}$ can also be written as a polynomial $P_{G}(\underline{t}, \underline{p})$, homogeneous of degree $b_{1}(G)+1$ in $\underline{t}$. The graph polynomial $\Psi_{G}(\underline{t})$ is also known as the first Symanzik polynomial and $P_{G}(\underline{t}, \underline{p})$ as the second Symanzik polynomial of the graph $G$. Up to a numerical factor including $C_{\ell, n}$ and powers of $2 \pi$, and up to a divergence captured by a Gamma factor $\Gamma(n-D \ell / 2)$, the parametric form of the Feynman integral $U(G)$ is then given by

$$
U(G)=\int_{\sigma_{n}} \frac{P_{G}(\underline{t}, \underline{p})^{-n+D \ell / 2}}{\Psi_{G}(\underline{t})^{-n+D(\ell+1) / 2}} \omega_{n}
$$

with $\omega_{n}$ the volume form on the simplex $\sigma_{n}$ induced by $d t_{1} \cdots d t_{n}$. We focus here on the range where $D \ell \geq 2 n$ so that the integrand is a rational function with the first Symanzik polynomial in the denominator. Modulo the important issue of infrared divergences caused by the intersections of 
the domain of integration $\sigma_{n}$ and the hypersurface defined by the vanishing $\Psi_{G}(\underline{t})=0$, which need to be dealt with through an appropriate renormalization procedure that we will not be discussing here, the parametric Feynman integral looks formally like a period of an algebraic differential form defined on the graph hypersurface complement $\check{Y}_{G}=\mathbb{A}^{n} \backslash \check{X}_{G}$. Thus, knowledge of the motivic properties of these graph hypersurface complements can predict the nature of the numbers that can occur as periods.

Extensive computational results of Broadhurst and Kreimer [39] found the systematic occurrence of multiple zeta values in such computations of Feynman integrals. Multiple zeta values can be realized as periods of 'mixed-Tate motives' [40] and in fact all periods of mixed-Tate motives are $\mathbb{Q}\left[(2 \pi i)^{-1}\right]$-linear combinations of multiple zeta values, [41]. The occurrence of multiple zeta values in Feynman integral computations originally motivated the conjecture that the varieties $\check{Y}_{G}$ would all be mixed-Tate motives. This was originally formulated by Kontsevich as a question on the polynomial summability of the graph hypersurfaces $X_{G}$, which can be also formulated as the question of whether the classes $\left[X_{G}\right]$ in the Grothendieck ring of varieties belong to the subring $\mathbb{Z}[\mathbb{L}]$ generated by the Lefschetz motive $\mathbb{L}=\left[\mathbb{A}^{1}\right]$. This conjecture was disproved by Belkale and Brosnan [42] who showed that the classes $\left[X_{G}\right]$ additively generate the localization of the Grothendieck ring obtained by inverting the classes $\left[\mathrm{GL}_{n}\right]$ for all $n$. This means that one expects to see arbitrarily complicated motives occurring when arbitrarily large graphs are considered. Specific counterexamples of non-mixed-Tate graph hypersurfaces, for the $\varphi^{4}$-scalar quantum field theory and for graphs starting at 14 edges, were found by Doryn [43] and by Schnetz [44]. It remains an interesting question to understand which motives occur and which families of graphs give rise to mixed-Tate motives.

Some related constructions of algebraic varieties associated to parametric Feynman integrals are worth mentioning. The first is a variant on the graph hypersurface $\check{X}_{G}$, which however is always a mixed-Tate motive for all graphs $G$. It is obtained in the following way. In the derivation of the parametric Feynman integral recalled above, instead of fixing the choice of the $u_{e}$ as a solution to the momentum conservation equation and $\sum_{e} t_{e} u_{e} \eta_{e, j}=0$, we can consider the $u_{e}$ as ranging over all possible solutions. These are all of the form

$$
u_{e}=\sum_{r=1}^{\ell} \eta_{e, r} \beta_{r},
$$

where the orthogonality constraint above becomes

$$
0=\sum_{e, r} t_{e} \eta_{e, r} \eta_{e, j} \beta_{r}=Q_{t}(\beta)
$$

This determines a complete intersection variety

$$
\Lambda_{G}=\left\{(t, \beta) \mid Q_{t}(\beta)=0\right\},
$$

with $\left(Q_{t}(\beta)\right)_{j}=\sum_{e, r} t_{e} \eta_{e, r} \eta_{e, j} \beta_{r}$. One obtains in this way a formulation of the Feynman integral where the relevant variety replacing the graph hypersurface complement $\check{X}_{G}$ is the variety $\Lambda_{G}$ defined above, see [45]. In terms of motivic properties, the varieties $\Lambda_{G}$ were introduced by Esnault and Bloch in relation to Hodge structures and shown to be always mixed-Tate, [46]. 
Another related construction, also due to Bloch [47], shows that, while the graph hypersurfaces $X_{G}$ themselves are not always mixed-Tate, if one considers a suitable combination of the classes $\left[X_{G}\right]$, obtained by summing over all graphs with fixed number of vertices, with each class $\left[X_{G}\right]$ weighted by a combinatorial symmetry factor $n ! /|A u t(G)|$, then the resulting Grothendieck class is always mixed-Tate. This observation is consistent with the fact that, in physics, it is not the contribution of individual Feynman integrals that has physical meaning, but the combined contribution, appropriately weighted by symmetry factors, of all graphs at a given order (usually loop number or number of internal edges) in the perturbative expansion.

We also should mention another important algebro-geometric construction in perturbative quantum field theory related to the parametric form of Feynman integrals. There are linear relations satisfied by Feynman integrals, which significantly reduce the combinatorial complexity of the perturbative expansion. The coefficients of these linear relations are rational functions of the spacetime dimension $D$, the mass parameters and the external momenta, [48]. This makes it possible to reduce, through an integration by parts method, the computation of Feynman integrals to a finite set of master integrals. It is shown in [49] that the number of master integrals can be computed as a vector space dimension, which in turn is computed by the Euler characteristic of a hypersurface complement. As in the previous discussion of parametric Feynman integrals, one writes the combination $\sum_{e} t_{e} q_{e}$ in the loop momentum variables $x_{i}(i=1, \ldots, \ell)$ as a quadratic, a linear, and a constant term

$$
\sum_{e} t_{e} q_{e}=\sum_{i, j} M_{i j} x_{i} x_{j}+\sum_{i} 2 Q_{i} x_{i}+J
$$

and set $\mathcal{G}=\mathcal{U}+\mathcal{F}$ with $\mathcal{U}=\operatorname{det} M$ and $\mathcal{F}=\mathcal{U}\left(Q^{t} M^{-1} Q+J\right)$, where

$$
\mathcal{U}^{(\ell+1) D / 2-|v|} \mathcal{F}^{|v|-\ell D / 2}
$$

recovers the same integrand of the parametric Feynman integral described above, where $\mathcal{U}$ and $\mathcal{F}$ occur as the first and second Symanzik polynomials, in the slightly more general form where we have exponents $v_{i}$ of the denominators $q_{i}$ and we use the more general version of the Feynman parameters formula given by

$$
\frac{1}{q_{1}^{\nu_{1}} \cdots q_{n}^{\nu_{n}}}=\frac{\Gamma(|v|)}{\Gamma\left(v_{1}\right) \cdots \Gamma\left(v_{n}\right)} \int_{[0,1]^{n}} \frac{\delta\left(1-\sum_{i} t_{i}\right)}{\left(t_{1} q_{1}+\cdots+t_{n} q_{n}\right)^{n}} t_{1}^{\nu_{1}-1} \cdots t_{n}^{\nu_{n}-1} d t_{1} \cdots d t_{n},
$$

with $|v|=v_{1}+\cdots+v_{n}$, so that the parametric Feynman integral is written, up to a numerical factor and Gamma functions, in the form

$$
U(G)=\int_{\sigma_{n}} \frac{\omega_{n}}{\mathcal{U}^{(\ell+1) D / 2-|v| \mathcal{F}|v|-\ell D / 2}}
$$

This can be equivalently reformulated in the Lee-Pomeransky form, where, with numerical and Gamma factors included

$$
U(G)=\frac{\Gamma(D / 2)}{\Gamma((\ell+1) D / 2-|v|)}\left(\prod_{i} \int_{\mathbb{R}} \frac{x_{i}^{v_{i}-1} d x_{i}}{\Gamma\left(v_{i}\right)}\right) \mathcal{G}^{-D / 2} .
$$


This formulation of the parametric Feynman integrals is a multidimensional Mellin transform of the function $\mathcal{G}^{-D / 2}$. If one considers only those relations between Feynman integrals that do not involve different graphs, so that one can work with a fixed $G$ and the corresponding $\mathcal{G}=\mathcal{G}_{G}$, then those relations that change the exponents $v_{i}$ by integer shifts can be identified, via Mellin transform, with differential operators that annihilate $\mathcal{G}^{-D / 2}$. These can be treated using results from the theory of $D$-modules. Consider the algebraic torus $\mathbb{G}_{m}^{n}$ and the hypersurface $V(\mathcal{G})=\left\{t=\left(t_{e}\right) \mid \mathcal{G}(t)=0\right\}$ inside the torus, $V(\mathcal{G}) \subset \mathbb{G}_{m}^{n}$, with

$$
O\left(\mathbb{G}_{m}^{n} \backslash V(\mathcal{G})\right)=\mathbb{C}\left[t_{e}^{ \pm 1}, \mathcal{G}^{-1}\right] .
$$

The space of all master integrals, seen as Mellin transforms of $\mathcal{G}^{s}$, can be identified with the $\mathbb{C}(s, v)$ vector space $V_{\mathcal{G}}$ spanned by the integrals with parameters $v_{i}$ shifted by an integer $n$. The number of master integrals is the dimension $\operatorname{dim}_{\mathbb{C}(s, v)} V_{\mathcal{G}}$. Using a result of Loeser and Sabbah, [50], [51], this dimension can be computed (see Section 3 of [49]) as the Euler characteristic of the algebraic de Rham complex of a holonomic $D$-module, which is then identified with the Euler characteristic of the algebraic de Rham complex of $\mathbb{C}\left[t_{e}^{ \pm 1}, \mathcal{G}^{-1}\right]$, hence the Euler characteristic of the hypersurface complement $\mathbb{G}_{m}^{n} \backslash V(\mathcal{G})$. It seems an interesting question what additional information on the space of master integrals can be derived from the additional information, beyond the Euler characteristic, given by the CSM characteristic classes of the hypersurface $V(\mathcal{G})$ and its complement $\mathbb{G}_{m}^{n} \backslash V(\mathcal{G})$.

\subsection{Algebro-geometric Feynman rules: motivic Feynman rules}

The 'Feynman rules' are a formalism that extracts from a given graph the corresponding contribution to the Feynman amplitude. The rules themselves satisfy an interesting general structure. For example, if $\langle G\rangle$ denotes the contribution of $G$ to an amplitude, then it can be shown that

$$
\sum_{\text {graphs } G}\langle G\rangle=\exp \left(\sum_{\text {connected graphs } G}\langle G\rangle\right) ;
$$

this equality encodes a crucial contribution of symmetries in evaluating $\langle G\rangle$ in terms of the product of the contributions of its connected components. In particular, we could restrict our attention to connected graphs. Further, if $G$ is obtained as the join of two subgraphs $G_{1}, G_{2}$ by a single edge, then

$$
\langle G\rangle=\text { 'propagator' } \cdot\left\langle G_{1}\right\rangle \cdot\left\langle G_{2}\right\rangle
$$

including a multiplicative factor (the '(inverse) propagator') that is independent of the graphs.

Some of this structure is mirrored by the graph polynomial itself. For example, it is easy to see that if $G$ is the disjoint union of two graphs $G_{1}$ and $G_{2}$ (including the possibility that $G_{1}$ and $G_{2}$ may be connected at a vertex), then $\Psi_{G}=\Psi_{G_{1}} \cdot \Psi_{G_{2}}$. We say that a ring-valued invariant $U(G)$ of a graph is an 'abstract Feynman rule' if $U(G)=U\left(G_{1}\right) U\left(G_{2}\right)$ when $G$ is obtained by joining $G_{1}$ and $G_{2}$ at a vertex. In particular, if $G_{1}$ and $G_{2}$ are joined by an edge $e$, then $U(G)=U(e) U\left(G_{1}\right) U\left(G_{2}\right)$ : thus, the invariant for a single edge behaves as an 'propagator' for these rules.

The reason why the very simple multiplicative property over disjoint union of graphs is referred to as an "abstract Feynman rule" lies in the fact that this is the minimal algebraic requirement needed to set up an "algebraic renormalization procedure". This requires the additional property that the 
target commutative ring $\mathcal{R}$ is a Rota-Baxter algebra of weight -1 , while the source polynomial algebra on the Feynman graphs (with product given by the disjoint union) is also endowed with a coproduct making it into a Hopf algebra $\mathcal{H}$. A Rota-Baxter algebra (ring) of weight $\lambda$ is a unital commutative algebra (ring) $\mathcal{R}$ endowed with a linear operator $\mathcal{T}: \mathcal{R} \rightarrow \mathcal{R}$ which satisfies the $\lambda$-Rota-Baxter identity

$$
\mathcal{T}(a) \mathcal{T}(b)=\mathcal{T}(a \mathcal{T}(b))+\mathcal{T}(\mathcal{T}(a) b)+\lambda \mathcal{T}(a b) .
$$

Note here an important point: the morphism $\phi: \mathcal{H} \rightarrow \mathcal{R}$ that we are referring to as an abstract Feynman rule is only a morphism of commutative algebras, and knows nothing about the coproduct of $\mathcal{H}$ and the Rota-Baxter operator of $\mathcal{R}$. However, the multiplicativity of $\phi$, together with the coproduct of $\mathcal{H}$ and the Rota-Baxter operator of $\mathcal{R}$ determine a unique multiplicative splitting (Birkhoff factorization) of $\phi$ into two algebra homomorphisms with respective targets given by subalgebras of $\mathcal{R}$ corresponding, respectively, to renormalized values and counterterms in the physical case. Indeed, the Rota-Baxter operator $\mathcal{T}$ determines a decomposition of $\mathcal{R}$ into two commutative algebras (rings), $\mathcal{R}_{+}=(1-\mathcal{T}) \mathcal{R}$ and $\mathcal{R}_{-}$the unitization of $\mathcal{T} \mathcal{R}$. The convolution product $\star$ of morphisms $\phi_{1}, \phi_{2} \in \operatorname{Hom}_{A l g}(\mathcal{H}, \mathcal{R})$ dual of the coproduct $\Delta$ of $\mathcal{H}$ is given by

$$
\phi_{1} \star \phi_{2}(x)=\left\langle\phi_{1} \otimes \phi_{2}, \Delta(x)\right\rangle=\sum \phi_{1}\left(x^{(1)}\right) \phi_{2}\left(x^{(2)}\right),
$$

for $\Delta(x)=\sum x^{(1)} \otimes x^{(2)}=x \otimes 1+1 \otimes x+\sum x^{\prime} \otimes x^{\prime \prime}$. Since $\mathcal{H}$ is a graded connected commutative Hopf algebra, the antipode is defined inductively by $S(x)=-x-\sum S\left(x^{\prime}\right) x^{\prime \prime}$. The Birkhoff factorization of an algebraic Feynman rule $\phi \in \operatorname{Hom}_{A l g}(\mathcal{H}, \mathcal{R})$ is defined inductively through the explicit formula

$$
\begin{gathered}
\phi_{-}(x)=-\mathcal{T}\left(\phi(x)+\sum \phi_{-}\left(x^{\prime}\right) \phi\left(x^{\prime \prime}\right)\right) \\
\phi_{+}(x)=(1-\mathcal{T})\left(\phi(x)+\sum \phi_{-}\left(x^{\prime}\right) \phi\left(x^{\prime \prime}\right)\right) .
\end{gathered}
$$

The Rota-Baxter identity for $\mathcal{T}$ ensures that these maps $\phi_{ \pm} \in \operatorname{Hom}_{A l g}\left(\mathcal{H}, \mathcal{R}_{ \pm}\right)$are indeed algebra homomorphisms.

The case where $\mathcal{R}$ is an algebra of Laurent power series, with projection onto the polar part as Rota-Baxter operator, corresponds to the physical case where the resulting renormalization procedure (the Connes-Kreimer renormalization) recovers the physical BPHZ renormalization procedure. The same structure however is implementable for other types of Rota-Baxter structure, hence we use in this setting the broader term "abstract Feynman rules" and "algebraic renormalization". We will not review here the detailed properties of Rota-Baxter algebras and Birkhoff factorization, as we will not need them in the rest of the paper, but we refer the readers to [52], [53], [54] for a detailed discussion of Connes-Kreimer renormalization. For examples of this formalism of algebraic renormalization applied outside of the quantum field theory BPHZ renormalization, with other Rota-Baxter structures, see for instance [55], [56].

As a broad class of examples of abstract Feynman rules, we can take the 'Tutte-Grothendieck invariants' of graphs, that is, specializations of the Tutte polynomial. Many important invariants of graphs (e.g., the chromatic and flow polynomials, the partition function of the Ising model, etc.) are invariants of this type. We note that Tutte-Grothendieck invariants satisfy, essentially by definition, simple formulas with respect to the operations of deletion $G \backslash e$ and contraction $G / e$ of an edge $e$ of 
a graph $G$. As a consequence, they also satisfy 'multiple edge formulas', which can be assembled into a generating function: if $T_{G}(x, y)$ denotes the Tutte polynomial of a graph $G$, and we denote by $G_{m e}$ the graph obtained by replacing an edge $e$ by $m$ parallel edges, then

$$
\sum_{m \geq 0} T_{G_{m e}}(x, y) \cdot \frac{s^{m}}{m !}=e^{s}\left(T_{G \backslash e}(x, y)+\frac{e^{(y-1) s}-1}{y-1} T_{G / e}(x, y)\right)
$$

provided that $e$ is not a bridge or a looping edge in $G$. While this tighter structure is not satisfied by arbitrary abstract Feynman rules, we will see that glimpse of it are satisfied by the rules that arise naturally in the context of graph hypersurfaces.

For us ([57, 58]), an 'algebro-geometric Feynman rule' is an abstract Feynman rule which only depends on the complement $\check{Y}_{G}:=\mathbb{A}^{n} \backslash \check{X}_{G}$. The dependence of an algebro-geometric Feynman rule on $\check{Y}_{G}$ does not a priori depend only on the isomorphism class of $\check{Y}_{G}$. For example, it may depend also on the specific data of immersed algebraic varieties, for which a suitable form of Grothendieck ring of immersed conical varieties can be defined, see [57]. We say that an algebro-geometric Feynman rule is 'motivic' if it only depends on the class $\mathbb{U}(G):=\left[\check{Y}_{G}\right]$ in the Grothendieck ring $K\left(\operatorname{Var}_{k}\right)$. In fact, the class $\mathbb{U}(G)$ is itself an algebro-geometric Feynman rule, with propagator given by the Lefschetz-Tate motive $\mathbb{L}$. Indeed, it is essentially straightforward to verify that if $G=G_{1} \amalg G_{2}$, then $\check{Y}_{G} \cong \check{Y}_{G_{1}} \times \check{Y}_{G_{2}}$ : therefore, $\mathbb{U}\left(G_{1} \amalg G_{2}\right)=\mathbb{U}\left(G_{1}\right) \mathbb{U}\left(G_{2}\right)$; and if $G=e$ consists of a single edge, then $\Psi_{G}=1$, so that

$$
\mathbb{U}(e)=\left[\mathbb{A}^{1} \backslash \emptyset\right]=\left[\mathbb{A}^{1}\right]=\mathbb{L} .
$$

Example 4.3. For the '3-banana' $G$ of Example 4.2,

$$
\mathbb{U}(G)=\mathbb{L}^{2}(\mathbb{L}-1)
$$

as may be checked easily: the complement $\check{Y}_{G}$ of the affine graph hypersurface maps to the complement of a smooth conic in $\mathbb{P}^{2}$, with $k^{*}$ fibers; smooth conics are isomorphic to $\mathbb{P}^{1}$, and the result follows.

This motivic Feynman rule is not a Tutte-Grothendieck invariant. This fact reflects the important observation, due to Belkale and Brosnan and already discussed in $\$ 4.2$, that in general graph hypersurfaces do not determine mixed-Tate motives, that is, polynomials in the class $\mathbb{L}$. If recursive formulas under deletion and contraction held for the motivic Feynman rule, it would follow that the class of every graph hypersurface is in $\mathbb{Z}[\mathbb{L}]$.

It is natural then to inquire to what extent a given algebro-geometric Feynman rule may satisfy deletion-contraction or multiple-edge formulas: for motivic Feynman rules, this may be viewed as a way to quantify the extent to which graph hypersurfaces fail to be mixed-Tate motives, and the recursive process underlying multiple-edge formulas may be used to provide families of graphs whose hypersurface do determine mixed-Tate motives. For instance, for certain SYK quantum field theories that are dominated by the melonic sector, one sees by a careful use of these recursive procedures that the dominant contributions to the asymptotic expansion remain in the mixed-Tate class, see [59].

The behavior of the motivic Feynman rule with respect to these operations amounts to the following statement. 
Theorem 4.1 ([58]). Let $\mathbb{U}(G):=\left[\check{Y}_{G}\right] \in K\left(\operatorname{Var}_{k}\right)$. Assume the edge e of $G$ is neither a bridge nor a looping edge. Then

$$
\mathbb{U}(G)=\mathbb{L} \cdot\left[\check{Y}_{G \backslash e} \cup \check{Y}_{G / e}\right]-\mathbb{U}(G \backslash e) .
$$

Further, let $G_{m e}$ be the graph obtained by replacing e with $m$ parallel edges. Then

$$
\mathbb{U}\left(G_{2 e}\right)=(\mathbb{L}-2) \cdot \mathbb{U}(G)+(\mathbb{L}-1) \cdot \mathbb{U}(G \backslash e)+\mathbb{L} \cdot \mathbb{U}(G / e) \quad ;
$$

and

$$
\begin{aligned}
\sum_{m \geq 0} \mathbb{U}\left(G_{m e}\right) \frac{s^{m}}{m !}=\frac{e^{(\mathbb{L}-1) s}-e^{-s}}{\mathbb{L}} \mathbb{U}(G)+\frac{e^{(\mathbb{L}-1) s}+(\mathbb{L}-1) e^{-s}}{\mathbb{L}} \mathbb{U}(G \backslash e) \\
+\left(s e^{(\mathbb{L}-1) s}-\frac{e^{(\mathbb{L}-1) s}-e^{-s}}{\mathbb{L}}\right) \mathbb{U}(G / e) .
\end{aligned}
$$

The first formula is the analogue for motivic Feynman rules of a deletion-contraction formula. Note that it includes a term, $\left[\check{Y}_{G \backslash e} \cup \check{Y}_{G / e}\right]$, which does not only depend on combinatorial information, in the sense that it is not the Grothendieck class of a graph hypersurface complement. In a sense, this term is responsible for the fact that graph hypersurfaces are not necessarily mixed-Tate, as it prevents a straightforward recursive determination of the class $\mathbb{U}(G)$. It is quite interesting that the second and third formulas are combinatorial, thus close in spirit with the situation for Tutte invariants. Compare the third formula with the corresponding formula (17) that holds for Tutte polynomials. The coefficients appearing in this expression are independently significant, as they are related to functions used in defining Hirzebruch's $T_{y}$ genus.

Example 4.4. The ' $n$-banana graph'

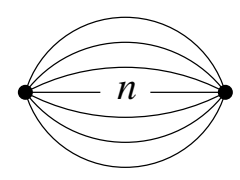

may be viewed as $G_{m e}$ for $m=n-2$, where $G$ is the 3-banana graph. We found $\mathbb{U}(G)=\mathbb{L}^{2}(\mathbb{L}-1)$ (Example 4.3), and it is easy to verify that

$$
\mathbb{U}(G \backslash e)=\mathbb{L}(\mathbb{L}-1) \quad, \quad \mathbb{U}(G / e)=(\mathbb{L}-1)^{2} .
$$

By Theorem 4.1, a generating function for the class $\mathbb{U}\left(G_{n}\right)$ is

$$
\sum_{n \geq 2} \mathbb{U}\left(G_{n}\right) \frac{s^{n-2}}{(n-2) !}=(\mathbb{L}-1)\left(\left(\mathbb{L}^{2} s-\mathbb{L} s+\mathbb{L}^{2}+1\right) \frac{e^{(\mathbb{L}-1) s}-e^{-s}}{\mathbb{L}}+(\mathbb{L} s+\mathbb{L}-s) e^{-s}\right)
$$

One may observe that this expression equals

$$
\frac{d}{d s^{2}}\left((\mathbb{L}-1) \frac{e^{(\mathbb{L}-1) s}-e^{-s}}{\mathbb{L}}+s e^{(\mathbb{L}-1) s}\right)
$$

and it follows that

$$
\mathbb{U}\left(G_{n}\right)=(\mathbb{L}-1) \frac{(\mathbb{L}-1)^{n}-(-1)^{n}}{\mathbb{L}}+n(\mathbb{L}-1)^{n-1}
$$

for $n \geq 2$. 


\subsection{Chern-Schwartz-MacPherson Feynman rules}

Recall that the Grothendieck ring of varieties acts as a 'universal Euler characteristic'. Thus, the formulas obtained in Theorem 4.1 hold for every additive and multiplicative ring-valued invariants of complements of affine graph hypersurfaces.

The Euler characteristic is such an invariant, but it is not useful in this case (as it equals 0 if the graph is not a forest); it is natural to inquire whether the other generalization of the Euler characteristic that we have examined in $\$ 3.2$, that is, the Chern-Schwartz-MacPherson class, may also be used to define abstract Feynman rules. The result of this last section in this paper is that this is indeed the case. Thus, the intersection theory (in the sense of §2) used in our treatment of characteristic classes for singular/noncompact varieties provides an alternative way of encoding some of the basic structure underlying amplitudes in perturbative quantum field theory.

The resulting graph-theoretic invariant is not motivic, that is, it is not a specialization of the motivic Feynman rules introduced above, and also is not a Tutte-Grothendieck invariant. In fact, its behavior with respect to deletion and contraction is very subtle, and we will close this paper by describing multiple-edge formulas that it satisfies.

Remark 4.1. The Euler characteristic of the complement $Y_{G}$ of the projective graph hypersurface $X_{G}$ carries more information than its affine counterpart; see below. Contrary to an early guess of the first author, the Euler characteristic $\chi\left(Y_{G}\right)$ can take any integer value, as has been proved recently ([60]).

In order to define the 'Chern-Schartz-MacPherson' Feynman rules, recall that in $\S 3.2$ we have defined a class $c_{\mathrm{SM}}(U) \in A_{*}(V)$ for every locally closed subset $U$ of a variety $V$. For a graph $G$ with $n$ edges we let $V=\mathbb{P}^{n}$, so that elements of the Chow group $A_{*}(V)$ may be written as a linear combination

$$
a_{0}\left[\mathbb{P}^{0}\right]+a_{1}\left[\mathbb{P}^{1}\right]+\cdots+a_{n}\left[\mathbb{P}^{n}\right]
$$

with integer coefficients $a_{i}$. We say that a locally closed subset $U \subseteq \mathbb{A}^{n}$ is 'conical' if $u \in U$ if and only if $\lambda u \in U$ for all $\lambda \in k^{*}$. With every locally closed conical subset $U \subseteq \mathbb{A}^{n}$ we associate the polynomial

$$
F_{U}(t):=a_{0}+a_{1} T+\cdots+a_{n} T^{n}
$$

determined by the coefficients $a_{i}$ in the class

$$
c_{\mathrm{SM}}(U)=a_{0}\left[\mathbb{P}^{0}\right]+a_{1}\left[\mathbb{P}^{1}\right]+\cdots+a_{n}\left[\mathbb{P}^{n}\right],
$$

where we view $U$ as a locally closed subset of the projective space $\mathbb{P}^{n}$ obtained by completing $\mathbb{A}^{n}$. Example 4.5. An affine subspace $\mathbb{A}^{k}$ through the origin in $\mathbb{A}^{n}$ is conical. Its completion in $\mathbb{P}^{n}$ is a subspace $\mathbb{P}^{k}$. By additivity, and denoting by $H$ the hyperplane class,

$$
\begin{aligned}
c_{\mathrm{SM}}\left(\mathbb{A}^{k}\right) & =c_{\mathrm{SM}}\left(\mathbb{P}^{k}\right)-c_{\mathrm{SM}}\left(\mathbb{P}^{k-1}\right) \\
& =c\left(T \mathbb{P}^{k}\right) \cap\left[\mathbb{P}^{k}\right]-c_{\mathrm{SM}}\left(T \mathbb{P}^{k-1}\right) \cap\left[\mathbb{P}^{k-1}\right] \\
& =\left((1+H)^{k+1} \cdot H^{n-k}-(1+H)^{k} \cdot H^{n-k+1}\right) \cap\left[\mathbb{P}^{n}\right] \\
& =(1+H)^{k} \cdot H^{n-k} \cap\left[\mathbb{P}^{n}\right] \\
& =\sum_{i=0}^{k}\left(\begin{array}{l}
k \\
i
\end{array}\right)\left[\mathbb{P}^{i}\right],
\end{aligned}
$$


therefore

$$
F_{\mathbb{A}^{k}}(T)=\sum_{i=0}^{k}\left(\begin{array}{l}
k \\
i
\end{array}\right) T^{i}=(1+T)^{k} .
$$

Note that the result is independent of the ambient dimension $n$.

It is in fact easy to see that if $U \subseteq \mathbb{A}^{n}$, and $\mathbb{A}^{n}$ is viewed as a subspace of $\mathbb{A}^{m}$ for some $m \geq n$, then $F_{U}(t)$ may be computed by using either embedding.

The following lemma is key for the considerations that follow.

Lemma 4.2. The invariant $F_{U}(T)$ is additive, in the sense that if $U^{\prime}, U^{\prime \prime}$ are locally closed conical subsets of $\mathbb{A}^{n}$, then

$$
F_{U^{\prime} \cup U^{\prime \prime}}(T)=F_{U^{\prime}}(T)+F_{U^{\prime \prime}}(T)-F_{U^{\prime} \cap U^{\prime \prime}}(T) .
$$

It is also multiplicative, in the following sense: Let $U^{\prime} \subseteq \mathbb{A}^{n^{\prime}}, U^{\prime \prime} \subseteq \mathbb{A}^{n^{\prime \prime}}$ be locally closed conical affine varieties. Then

$$
F_{U^{\prime} \times U^{\prime \prime}}(T)=F_{U^{\prime}}(T) \cdot F_{U^{\prime \prime}}(T)
$$

The first part of Lemma 4.2 follows from the additivity property of Chern-Schwartz-MacPherson classes, cf. (11). The second part of the lemma is perhaps subtler than it looks. If $U^{\prime} \subseteq \mathbb{A}^{n}$ and $U^{\prime \prime} \subseteq \mathbb{A}^{m}$, then the left-hand side refers to a CSM class computed in $\mathbb{P}^{m+n}$. Standard product formulas for CSM classes reduce the right-hand side to a computation for a locally closed subset of $\mathbb{P}^{m} \times \mathbb{P}^{n}$. Comparing classes in these two different completions of $\mathbb{A}^{m+n}$ requires some work, carried out in [57]. An alternative approach, pointed out by Schürmann and by Weber ([32]), interprets the polynomial $F_{U}(T)$ in terms of equivariant Chern-Schwartz-MacPherson classes, and the product formula is straightforward from this point of view.

Lemma 4.2 justifies the following definition. Recall that for a graph $G$ with $n$ edges, $\check{Y}_{G}$ denotes the complement $\mathbb{A}^{n} \backslash \check{X}_{G}$ of the affine hypersurface defined by the graph polynomial $\Psi_{G}$.

Definition 4.1. The Chern-Schwartz-MacPherson Feynman rules assign to every graph $G$ the polynomial

$$
C_{G}(T)=F_{\check{Y}_{G}}(T)
$$

in $\mathbb{Z}[T]$.

By Lemma 4.2,

$$
C_{G}(T)=C_{G_{1}}(T) \cdot C_{G_{2}}(T)
$$

if $G$ consists of the disjoint union of $G_{1}$ and $G_{2}$, possibly joined at a single vertex. Thus the Chern-Schwartz-MacPherson Feynman rules are abstract (and algebro-geometric) Feynman rules in the sense introduced in $\S 4.3$. The propagator is $C_{e}(T)=F_{\mathbb{A}^{1}}(T)=1+T$. Comparing with the motivic propagator $\mathbb{U}(e)=\mathbb{L}$, it is tempting to view the variable $T$ as somehow related with the 'torus' $k^{*}$, whose Grothendieck class is $\mathbb{L}-1$.

Example 4.6. Explicit computations may be carried out in several cases using the methods summarized in 3.3, and are aided by properties of Chern-Schwartz-MacPherson Feynman rules that 
we will list below. For the $n$-banana graph $G_{n}$ of Example 4.4, one obtains the remarkably simple expression

$$
C_{G_{n}}(T)=T(T-1)^{n-1}+n T^{n-1} .
$$

This expression should be compared, both for similarities and differences, with (19). In particular, note that a simple substitution such as $T=\mathbb{L}-1$ does not lead to a match of these expressions.

We collect below several properties of the new polynomial graph invariant. Most follow easily from the definition and from Lemma 4.2.

- The polynomial $C_{G}(T)$ is of the form

$$
T^{n}+\left(n-b_{1}(G)\right) T^{n-1}+\text { lower degree terms , }
$$

where $n$ is the number of edges of $G$.

- $C_{G^{\prime}}(T)=(1+T) C_{G}(T)$ if $G^{\prime}$ is obtained from $G$ by splitting an edge or attaching an edge to a single vertex.

- $C_{G^{\prime}}(T)=T C_{G}(T)$ if $G^{\prime}$ is obtained from $G$ by attaching a looping edge to a vertex.

- $C_{G}(0)=1$ if $G$ is a forest. In fact, $C_{G}(T)=(1+T)^{n}$ if $G$ is a forest with $n$ edges; in particular

- $C_{G}(0)=0$ if $G$ is not a forest.

- $C_{G}^{\prime}(0)=\chi\left(Y_{G}\right)$, the Euler characteristic of the projective graph hypersurface complement.

Example 4.7. Let $G_{n}$ be an $n$-sided polygon. Then $G_{n}$ may be obtained by splitting the single edge of a looping edge $n-1$ times, and it follows that $C_{G_{n}}(T)=T(T+1)^{n-1}$.

This may of course be verified directly: the graph polynomial for a polygon is $t_{1}+\cdots+t_{n}$, therefore $\check{Y}_{G_{n}}=\mathbb{A}^{n}-\mathbb{A}^{n-1}$, hence

$$
C_{G_{n}}(T)=F_{\mathbb{A}^{n}-\mathbb{A}^{n-1}}(T)=F_{\mathbb{A}^{n}}(T)-F_{\mathbb{A}^{n-1}}(T)=(1+T)^{n}-(1+T)^{n-1},
$$

where we used the first part of Lemma 4.2 and the result obtained in Example 4.5.

Example 4.8. Let $G_{n}$ again be the $n$-banana graph. Then the Euler characteristic of the graph hypersurface $X_{G_{n}}$ defined by the Kirchhoff-Tutte-Symanzik polynomial of $G_{n}$ (whose complement in $\mathbb{P}^{n-1}$ is $\left.Y_{G_{n}}\right)$ is

$$
\chi\left(X_{G_{n}}\right)=n+(-1)^{n}
$$

for $n \geq 2$. (Indeed, $\chi\left(Y_{G_{n}}\right)=C_{G_{n}}^{\prime}(0)=(-1)^{n-1}$ according to (20).)

The equality $\chi\left(Y_{G}\right)=C_{G}^{\prime}(0)$ follows from a more refined result, relating $C_{G}(T)$ directly with the Chern-Schwartz-MacPherson class of the projective complement $Y_{G}=\mathbb{P}^{n-1} \backslash X_{G}$. Namely, one may verify that if

$$
C_{G}(T)=a_{0}+a_{1} T+\cdots+a_{n} T^{n},
$$

then

$$
c_{\mathrm{SM}}\left(Y_{G}\right)=a_{1}\left[\mathbb{P}^{0}\right]+\cdots+a_{n} T^{n-1}
$$


Thus, the coefficient of $T$ in $C_{G}(T)$, that is, $a_{1}=C_{G}^{\prime}(0)$, equals $\int c_{\mathrm{SM}}\left(Y_{G}\right)=\chi\left(Y_{G}\right)$ by the singular/noncompact Poincaré-Hopf theorem (cf. (12)).

Summarizing, if $G$ is not a forest, then

$$
C_{G}(T)=\chi\left(Y_{G}\right) T+\cdots+\left(n-b_{1}(G)\right) T^{n-1}+T^{n} .
$$

Incidentally, this expression along with Lemma 4.2 clarifies in what sense the Euler characteristic of the projective complement $Y_{G}$ fails to be multiplicative on disjoint unions, i.e., to be an abstract Feynman rule: if $G=G^{\prime} \amalg G^{\prime \prime}$ (and neither $G^{\prime}$ nor $G^{\prime \prime}$ are forests), then

$$
C_{G}(T)=C_{G^{\prime}}(T) C_{G^{\prime \prime}}(T)=\chi\left(Y_{G^{\prime}}\right) \chi\left(Y_{G^{\prime \prime}}\right) T^{2}+\cdots \quad:
$$

while $\chi\left(Y_{G}\right)=0$, since this equals the coefficient of $T$ in $C_{G}(T)$, the information of the product $\chi\left(Y_{G^{\prime}}\right) \chi\left(Y_{G^{\prime \prime}}\right)$ is preserved in the Chern-Schwartz-MacPherson Feynman rules, as the coefficient of $T^{2}$.

All coefficients in (22) may be interpreted in terms of Euler characteristics. We do not have a reference for the following result, so we will provide a proof.

Proposition 4.3. Let $G$ be a graph that is not a forest, and let

$$
C_{G}(T)=a_{1} T+a_{2} T^{2}+\cdots+a_{n} T^{n} .
$$

Denote by $e_{i}, i=0, \ldots, n-1$, the topological Euler characteristic of the intersection of $Y_{G}$ with a general linear subspace of codimension $i$ in $\mathbb{P}^{n-1}$. Then $a_{1}=e_{0}$ and

$$
a_{k}=\sum_{i=k-1}^{n-1}\left(\begin{array}{l}
i-1 \\
k-2
\end{array}\right) e_{i}
$$

for $k=2, \ldots, n$,

$$
e_{k}=\sum_{i=k+1}^{n}\left(\begin{array}{l}
i-2 \\
k-1
\end{array}\right)(-1)^{i-k-1} a_{i}
$$

for $k=1, \ldots, n-1$.

Proof. As pointed out in (21), the coefficients of $C_{G}(T)$ determine $c_{\mathrm{SM}}\left(Y_{G}\right)$. We assemble these coefficients in the polynomial

$$
\gamma(t):=\sum_{k=1}^{n} a_{k} t^{k-1} .
$$

We also assemble the Euler characteristics of general linear sections in a polynomial:

$$
\chi(t):=\sum_{k=0}^{n-1} \chi\left(Y_{G} \cap L_{k}\right) \cdot(-t)^{k}
$$

where $L_{k}$ is a general linear subspace $\mathbb{P}^{n-1-k}$ of codimension $k$. (So, for instance, the constant term of $\chi(t)$ equals $\chi\left(Y_{G}\right)$.) By [28, Theorem 1.1], the polynomials $\gamma(t)$ and $\chi(t)$ are interchanged by the involution

$$
p(t) \mapsto \frac{t \cdot p(-t-1)+p(0)}{t+1}
$$

This statement implies the two given expressions. 
The last issue we want to discuss is whether the Chern-Schwartz-MacPherson Feynman rules satisfy deletion-contraction or multiple edge formulas analogous to (17) (for Tutte-Grothendieck invariants) or the formula given in Theorem 4.1 (for the motivic Feynman rules).

Determining general deletion-contraction formulas for Chern-Schwartz-MacPherson Feynman rules is in fact an open question. Let $G$ be a graph, and let $e$ be an edge of $G$; we assume that $e$ is 'regular', that is, it is not a bridge or a looping edge of $G$, and $G \backslash e$ is not a forest. (These excluded cases are easy to treat separately.) We have a precise result if $G$ and $e$ satisfy two technical conditions, which we summarize as follows.

- Condition I: The Kirchhoff-Tutte-Symanzik graph polynomial $\Psi_{G}$ belongs to the Jacobian ideal of $\Psi_{G \backslash e}$.

- Condition II: The proper transform of $X_{G} \subseteq \mathbb{P}^{n-1}$ intersects transversally the exceptional divisor in a suitable blow-up.

The reader may find a more explicit statement of these conditions in [61, §2]. These conditions are needed in order to control the relevant intersection theory in a blow-up construction that relates the graph hypersurface of $G$ with the graph hypersurfaces of the deletion $G \backslash e$ and contraction $G / e$.

Theorem 4.4. Assume $G$ is a graph, and $e$ is a regular edge of $G$ for which conditions I and II hold. Then

$$
C_{G}(T)=F_{\check{Y}_{G \backslash e} \cup \check{Y}_{G / e}}(T)+(T-1) C_{G \backslash e}(T)
$$

Further

$$
C_{G_{2 e}}(T)=(2 T-1) C_{G}(T)-T(T-1) C_{G \backslash e}(T)+C_{G / e}(T) .
$$

Just as in the analogue for the motivic Feynman rules, stated in Theorem 4.1, the first formula includes an unavoidable term that is not determined by a single graph hypersurface.

We do not know of a combinatorial translation of the two technical conditions on which this statement relies; the first condition is studied from a combinatorial perspective in [62], but without reaching a characterization. There is however one case where the conditions automatically hold. The following is [61, Lemma 2.3].

Lemma 4.5. Let $G$ be a graph and let e be a regular edge of $G$. Assume that e has parallel edges in $G$. Then conditions I and II hold for $G$, $e$.

Therefore, we can give a multiple edge formula that does not rely on the technical conditions listed above.

Corollary 4.6. Let $G$ be a graph and let e be a regular edge of $G$. Then for $m \geq 1$

$$
C_{G_{(m+3) e}}(T)=(3 T-1) C_{G_{(m+2) e}}(T)-\left(3 T^{2}-2 T\right) C_{G_{(m+1) e}}(T)+\left(T^{3}-T^{2}\right) C_{G_{m e}}(T) .
$$

Example 4.9. Coming back once again to the example of $n$-bananas, (20), the recursion in Corollary 4.6 implies the identity

$$
\begin{aligned}
\left(T(T-1)^{m+2}+\right. & \left.(m+3) T^{m+2}\right)=(3 T-1)\left(T(T-1)^{m+1}+(m+2) T^{m+1}\right) \\
& -\left(3 T^{2}-2 T\right)\left(T(T-1)^{m}+(m+1) T^{m}\right)+\left(T^{3}-T^{2}\right)\left(T(T-1)^{m-1}+m T^{m-1}\right) .
\end{aligned}
$$


In fact, an explicit computation of the Chern-Schwartz-MacPherson Feynman rules for the 2-banana, the 3-banana, and the 4-banana suffice in order to obtain the general formula (20), by means of Corollary 4.6.

\section{Epilogue}

We have reviewed the apparatus of modern intersection theory in algebraic geometry and discussed an application in the form of a theory of 'characteristic classes' for possibly singular, possibly noncompact algebraic varieties. We have further illustrated this application by means of the example of graph hypersurfaces, whose study is motivated by the structure of perturbative quantum field theory.

We hope that this excursion will raise the reader's interest in this aspect of algebraic geometry. Whether this can be useful in the study of Feynman amplitudes in the style of the other talks in the MathemAmplitudes workshop will stand as an open question. Can one construct a 'CSM class' in twisted cohomology? Is there a good notion of 'twisted' algebro-geometric Feynman rules? Do relations among $c_{\mathrm{SM}}$ classes of relevant loci imply relations of corresponding Feynman amplitudes?

\section{Acknowledgments}

We thank the organizers of the Padova 2019 meeting on Intersection Theory and Feynman Integrals for the invitation to participate and for a very stimulating conference. The first author acknowledges support from a Simons Foundation Collaboration Grant, award number 625561, and thanks Caltech for the hospitality. The second author is partially supported by NSF grant DMS1707882, and by NSERC Discovery Grant RGPIN-2018-04937 and Accelerator Supplement grant RGPAS-2018-522593.

\section{References}

[1] W. Fulton, Intersection theory, Springer-Verlag, Berlin (1984).

[2] D. Eisenbud and J. Harris, 3264 and all that-a second course in algebraic geometry, Cambridge University Press, Cambridge (2016).

[3] P. Mastrolia and S. Mizera, Feynman integrals and intersection theory, J. High Energy Phys. (2019) 139 , front matter +24 .

[4] H. Frellesvig, F. Gasparotto, S. Laporta, M.K. Mandal, P. Mastrolia, L. Mattiazzi et al., Decomposition of Feynman integrals on the maximal cut by intersection numbers, J. High Energy Phys. (2019) 153, 110.

[5] H. Frellesvig, F. Gasparotto, M.K. Mandal, P. Mastrolia, L. Mattiazzi and S. Mizera, Vector space of Feynman integrals and multivariate intersection numbers, Phys. Rev. Lett. 123 (2019) 201602, 7.

[6] P. Aluffi, On some characteristic numbers for smooth plane curves (1987). 
[7] S.L. Kleiman and R. Speiser, Enumerative geometry of nonsingular plane cubics, in Algebraic geometry: Sundance 1988, vol. 116 of Contemp. Math., pp. 85-113, Amer. Math. Soc., Providence, RI (1991).

[8] R. Vakil, The characteristic numbers of quartic plane curves, Canad. J. Math. 51 (1999) 1089.

[9] A. Vistoli, Intersection theory on algebraic stacks and on their moduli spaces, Invent. Math. 97 (1989) 613.

[10] E. Looijenga, Intersection theory on Deligne-Mumford compactifications (after Witten and Kontsevich), No. 216, pp. Exp. No. 768, 4, 187-212 (1993).

[11] C. Faber, A conjectural description of the tautological ring of the moduli space of curves, in Moduli of curves and abelian varieties, Aspects Math., E33, pp. 109-129, Friedr. Vieweg, Braunschweig (1999).

[12] S. Keel, Intersection theory of moduli space of stable n-pointed curves of genus zero, Trans. Amer. Math. Soc. 330 (1992) 545.

[13] A. Gibney, S. Keel and I. Morrison, Towards the ample cone of $\bar{M}_{g, n}$, J. Amer. Math. Soc. 15 (2002) 273.

[14] P. Candelas, X.C. de la Ossa, P.S. Green and L. Parkes, A pair of Calabi-Yau manifolds as an exactly soluble superconformal theory, Nuclear Phys. B 359 (1991) 21.

[15] M. Kontsevich and Y. Manin, Gromov-Witten classes, quantum cohomology, and enumerative geometry, Comm. Math. Phys. 164 (1994) 525.

[16] W. Fulton and R. Pandharipande, Notes on stable maps and quantum cohomology, in Algebraic geometry-Santa Cruz 1995, vol. 62 of Proc. Sympos. Pure Math., pp. 45-96, Amer. Math. Soc., Providence, RI (1997).

[17] R. Pandharipande, Rational curves on hypersurfaces (after A. Givental), No. 252, pp. Exp. No. 848, 5, 307-340 (1998).

[18] B. Siebert, Virtual fundamental classes, global normal cones and Fulton's canonical classes, in Frobenius manifolds, Aspects Math., E36, pp. 341-358, Friedr. Vieweg, Wiesbaden (2004).

[19] D. Edidin and W. Graham, Equivariant intersection theory, Invent. Math. 131 (1998) 595.

[20] M. Esole, P. Jefferson and M.J. Kang, Euler characteristics of crepant resolutions of Weierstrass models, Comm. Math. Phys. 371 (2019) 99.

[21] G. Kennedy, MacPherson's Chern classes of singular algebraic varieties, Comm. Algebra 18 (1990) 2821. 
[22] P. Aluffi, Limits of Chow groups, and a new construction of Chern-Schwartz-MacPherson classes, Pure Appl. Math. Q. 2 (2006) 915.

[23] R.D. MacPherson, Chern classes for singular algebraic varieties, Ann. of Math. (2) 100 (1974) 423.

[24] M.-H. Schwartz, Classes caractéristiques définies par une stratification d'une variété analytique complexe. I, C. R. Acad. Sci. Paris 260 (1965) 3262.

[25] M.-H. Schwartz, Classes caractéristiques définies par une stratification d'une variété analytique complexe. II, C. R. Acad. Sci. Paris 260 (1965) 3535.

[26] J.-P. Brasselet and M.-H. Schwartz, Sur les classes de Chern d'un ensemble analytique complexe, in The Euler-Poincaré characteristic (French), vol. 83 of Astérisque, (Paris), pp. 93-147, Soc. Math. France (1981).

[27] P. Aluffi and J.-P. Brasselet, Une nouvelle preuve de la concordance des classes définies par M.-H. Schwartz et par R. MacPherson, Bull. Soc. Math. France 136 (2008) 159.

[28] P. Aluffi, Euler characteristics of general linear sections and polynomial Chern classes, Rend. Circ. Mat. Palermo (2) 62 (2013) 3.

[29] P. Aluffi, Chern classes for singular hypersurfaces, Trans. Amer. Math. Soc. 351 (1999) 3989.

[30] P. Aluffi, The Chern-Schwartz-MacPherson class of an embeddable scheme, Forum Math. Sigma 7 (2019) Paper No. e30, 28.

[31] T. Ohmoto, Equivariant Chern classes of singular algebraic varieties with group actions, Math. Proc. Cambridge Philos. Soc. 140 (2006) 115.

[32] A. Weber, Equivariant Chern classes and localization theorem, J. Singul. 5 (2012) 153.

[33] J.-P. Brasselet, J. Schürmann and S. Yokura, Hirzebruch classes and motivic Chern classes for singular spaces, J. Topol. Anal. 2 (2010) 1.

[34] R. Rimányi and A. Varchenko, Equivariant Chern-Schwartz-MacPherson classes in partial flag varieties: interpolation and formulae, in Schubert varieties, equivariant cohomology and characteristic classes-IMPANGA 15, EMS Ser. Congr. Rep., pp. 225-235, Eur. Math. Soc., Zürich (2018).

[35] P. Aluffi, L.C. Mihalcea, J. Schürmann and C. Su, "Shadows of characteristic cycles, Verma modules, and positivity of Chern-Schwartz-MacPherson classes of Schubert cells." arXiv:1709.08697.

[36] E. Looijenga, Motivic measures, Astérisque (2002) 267.

[37] F. Bittner, The universal Euler characteristic for varieties of characteristic zero, Compos. Math. 140 (2004) 1011. 
[38] P. Aluffi, Celestial integration, stringy invariants, and Chern-Schwartz-MacPherson classes, in Real and complex singularities, Trends Math., (Basel), pp. 1-13, Birkhäuser (2007).

[39] D.J. Broadhurst and D. Kreimer, Association of multiple zeta values with positive knots via Feynman diagrams up to 9 loops, Phys. Lett. B 393 (1997) 403.

[40] A.B. Goncharov and Y.I. Manin, Multiple $\zeta$-motives and moduli spaces $\overline{\mathscr{M}}_{0, n}$, Compos. Math. 140 (2004) 1.

[41] F. Brown, Mixed Tate motives over $\mathbb{Z}$, Ann. of Math. (2) 175 (2012) 949.

[42] P. Belkale and P. Brosnan, Matroids, motives, and a conjecture of Kontsevich, Duke Math. J. 116 (2003) 147.

[43] D. Doryn, On one example and one counterexample in counting rational points on graph hypersurfaces, Lett. Math. Phys. 97 (2011) 303.

[44] O. Schnetz, Quantum field theory over $\mathbb{F}_{q}$, Electron. J. Combin. 18 (2011) Paper 102, 23.

[45] D. Bejleri and M. Marcolli, Quantum field theory over $\mathbb{F}_{1}$, J. Geom. Phys. 69 (2013) 40.

[46] S. Bloch, "A note on hodge structures associated to graphs." https://www . math. uchicago.edu/ bloch/graph_hs090325.pdf.

[47] S. Bloch, Motives associated to sums of graphs, in The geometry of algebraic cycles, vol. 9 of Clay Math. Proc., pp. 137-143, Amer. Math. Soc., Providence, RI (2010).

[48] V.A. Smirnov, Analytic tools for Feynman integrals, vol. 250 of Springer Tracts in Modern Physics, Springer, Heidelberg (2012).

[49] T. Bitoun, C. Bogner, R.P. Klausen and E. Panzer, Feynman integral relations from parametric annihilators, Lett. Math. Phys. 109 (2019) 497.

[50] F. Loeser and C. Sabbah, Caractérisation des $\mathscr{D}$-modules hypergéométriques irréductibles sur le tore, C. R. Acad. Sci. Paris Sér. I Math. 312 (1991) 735.

[51] F. Loeser and C. Sabbah, Caractérisation des $\mathscr{D}$-modules hypergéométriques irréductibles sur le tore. II, C. R. Acad. Sci. Paris Sér. I Math. 315 (1992) 1263.

[52] A. Connes and D. Kreimer, Renormalization in quantum field theory and the Riemann-Hilbert problem. I. The Hopf algebra structure of graphs and the main theorem, Comm. Math. Phys. 210 (2000) 249.

[53] A. Connes and M. Marcolli, Noncommutative geometry, quantum fields and motives, vol. 55 of American Mathematical Society Colloquium Publications, American Mathematical Society, Providence, RI; Hindustan Book Agency, New Delhi (2008).

[54] M. Marcolli, Feynman motives, World Scientific Publishing Co. Pte. Ltd., Hackensack, NJ (2010). 
[55] Y.I. Manin and M. Marcolli, Quantum statistical mechanics of the absolute Galois group, SIGMA Symmetry Integrability Geom. Methods Appl. 16 (2020) Paper No. 038, 52.

[56] M. Marcolli and N. Tedeschi, Entropy algebras and Birkhoff factorization, J. Geom. Phys. 97 (2015) 243.

[57] P. Aluffi and M. Marcolli, Algebro-geometric Feynman rules, Int. J. Geom. Methods Mod. Phys. 8 (2011) 203.

[58] P. Aluffi and M. Marcolli, Feynman motives and deletion-contraction relations, in Topology of algebraic varieties and singularities, vol. 538 of Contemp. Math., (Providence, RI), pp. 21-64, Amer. Math. Soc. (2011).

[59] P. Aluffi, M. Marcolli and W. Qaisar, "Motives of melonic graphs." arXiv:2007.08565.

[60] G. Denham, D. Pol, M. Schulze and U. Walther, "Graph hypersurfaces with torus action and a conjecture of aluffi." arXiv:2005.02673, 2020.

[61] P. Aluffi, Chern classes of graph hypersurfaces and deletion-contraction relations, Mosc. Math. J. 12 (2012) 671.

[62] A. Kulkarni, G. Maxedon and K. Yeats, Some results on an algebro-geometric condition on graphs, J. Aust. Math. Soc. 104 (2018) 218. 